\title{
Value Iteration and Adaptive Dynamic Programming for Data-driven Adaptive Optimal Control Design
}

\author{
Tao Bian, Zhong-Ping Jiang, \\ Control and Networks Lab, Department of Electrical and Computer Engineering, Tandon School of Engineering, New York \\ University, 5 Metrotech Center, Brooklyn, NY 11201, USA
}

\begin{abstract}
This paper presents a novel non-model-based, data-driven adaptive optimal controller design for linear continuous-time systems with completely unknown dynamics. Inspired by the stochastic approximation theory, a continuous-time version of the traditional value iteration (VI) algorithm is presented with rigorous convergence analysis. This VI method is crucial for developing new adaptive dynamic programming methods to solve the adaptive optimal control problem and the stochastic robust optimal control problem for linear continuous-time systems. Fundamentally different from existing results, the $a$ priori knowledge of an initial admissible control policy is no longer required. The efficacy of the proposed methodology is illustrated by two examples and a brief comparative study between VI and earlier policy-iteration methods.
\end{abstract}

Key words: Value iteration, Adaptive dynamic programming, Optimal control, Adaptive control, Stochastic approximation.

\section{Introduction}

Dynamic programming (DP) (Bellman, 1957) is an approach to solving optimal control problems for dynamic systems using Bellman's principle of optimality. However, the implementation of traditional DP methods in real-world applications is prohibited due to the "curse of dimensionality" (Bellman, 1961) and the "curse of modeling" (Bertsekas and Tsitsiklis, 1996). Approximate DP and neuro-DP were introduced to conquer these two shortcomings by approximating the value function and control policy via on-line learning. In the past few decades, numerous approximate DP methods have been developed to solve the optimal control problem for Markov decision processes (Barto et al., 1983; Sutton, 1988; Watkins and Dayan, 1992; Tsitsiklis, 1994; Bertsekas, 2011). The interested reader can consult the nice tutorials by Bertsekas and Tsitsiklis (1996); Sutton and Barto (1998); and Si et al. (2004). Despite their popularity in real-world applications, the

\footnotetext{
* This paper was not presented at any IFAC meeting. Corresponding author T. Bian. Tel. +1-718-260-3779. Fax +1718-260-3906.

${ }^{\star \star}$ This work was partially supported by the National Science Foundation under Grants ECCS-1230040 and ECCS1501044.

Email addresses: tbian@nyu.edu (Tao Bian), zjiang@nyu.edu (Zhong-Ping Jiang).
}

above-mentioned methods usually overlooked the stability issue of the system. Moreover, the underlying state space is assumed either finite or countable, which is not always applicable in many applications.

Different from the early approximate DP methods, a new method known as adaptive dynamic programming (ADP), or heuristic dynamic programming in some literature, is introduced recently to find stabilizing optimal controllers for continuous-state space control systems via online learning. Over the past decade, ADP methods for discrete-time systems have attracted considerable attention of many researchers; see, Prokhorov and Wunsch (1997); Zhang et al. (2009); Lewis and Vamvoudakis (2011); Wang et al. (2011, 2012); Heydari (2014); Ni et al. (2015), and references therein. In parallel, the research in ADP for continuous-time systems is developed in Murray et al. (2002); Vrabie et al. (2009); Lewis and Vrabie (2009); Lewis et al. (2012); Vrabie et al. (2013); Xu et al. (2012); Jiang and Jiang (2012, 2013), and numerous references therein. For more recent work on continuoustime ADP, the interested reader may find more detailed references in Jiang and Jiang (2014b); Bian et al. (2014); Modares and Lewis (2014); Li et al. (2014); Bian et al. (2015); Song et al. (2015); Zargarzadeh et al. (2015); Bian et al. (2016).

Since existing continuous-time ADP methods are established based on policy iteration (PI) techniques (Leake 
and Liu, 1967; Kleinman, 1968; Beard et al., 1997), a common assumption is that a stabilizing control policy is known to start the learning process. However, this assumption is quite strong. When the system model is not fully accessible, finding an initial stabilizing control policy usually involves solving either a linear matrix inequality (LMI) (Jeung et al., 1996) or some matrix Riccati equations (Zhou and Khargonekar, 1988; Khargonekar et al., 1990), which is computationally expensive. Besides, since the bounds on the system parameters are often assumed known in these methods (which may be exploited to find the initial stabilizing control policy), the system model is not necessarily fully unknown.

In this paper, we depart from the commonly used PI scheme, and propose a new continuous-time VI algorithm that leads to the development of two ADP methods for linear continuous-time, continuous-state space systems. Both the optimal control and stochastic robust optimal control problems will be studied. Employing the VI method has at least two significant advantages: (1) an initial stabilizing control policy is not required; and (2) there is no need to solve matrix equation per-iteration. Due to these two advantages, VI has become the most widely used and best understood algorithm for solving discounted Markov decision problems (Puterman, 1994). Furthermore, VI method for discretetime, continuous-state space systems can also be found in Lancaster and Rodman (1995, Section 17.5) and Bertsekas (2005, Proposition 4.4.1), for the setting of linear systems; and in Liu et al. (2012), for a nonlinear extension. Unfortunately, VI methods for continuous-time, continuous-state space systems are still not well established. In Vrabie et al. (2013), some efforts have been made to derive a continuous-time VI algorithm, but the convergence of Vrabie's VI has not been proved.

Different from the past results, the continuous-time VI method given in this paper is inspired by the asymptotic stability property of the differential matrix Riccati equation (DMRE). It has been pointed out in Willems (1971); Kučera (1973); Shayman (1986) that under observability and stabilizability assumptions, the unique symmetric positive definite solution to the algebraic Riccati equation (ARE) is locally asymptotically stable (LAS) for the DMRE, backward in time. Since it is not easy to implement DMRE in ADP design, we borrow the idea of stochastic approximation to construct an iterative updating scheme based on the DMRE, and then use stochastic approximation method to show the convergence. While the stochastic approximation method used in this paper is inspired by the methods in Abounadi et al. (2002); Andrieu et al. (2005); Chen and Zhu (1986), it is slightly different from these results in the sense that the solution to the proposed algorithm stays in a subset of a level set of the Lyapunov function (see Lemma 3.4).

Since VI is employed in our ADP design, instead of starting from an initial stabilizing control policy, the pro- posed algorithms start from an arbitrary real symmetric and positive definite matrix representing the initial value function. Moreover, since the data matrices $(\Theta$ in (19) and $\Pi$ in (24)) in the ADP algorithms are independent of the number of learning iterations, there is no need to recalculate the matrix inverse in each iteration. This implies that our methods, for some systems, may be more computationally efficient in each iteration than the methods in Vrabie et al. (2009); Jiang and Jiang (2012) (see Remark 4.1 and Section 6.3). The obtained results are first tested by a single machine-infinite bus power system. Then, we test our stochastic robust ADP method with a human arm movement task (Wolpert et al., 2011). A comparison between our continuous-time VI and Kleinman's algorithm is also given. These examples show that our method serves as a powerful tool to solve non-model-based adaptive optimal control problems.

The remainder of this paper is organized as follows. In Section 2, some preliminaries regarding the optimal control problem for linear continuous-time systems are introduced. In Section 3, a new continuous-time VI method is presented with rigorous convergence analysis. Moreover, a detailed comparison between Vrabie's VI and our method is given. Based on the obtained result, two ADP methods for deterministic linear continuoustime systems are developed in Section 4. In Section 5, the obtained ADP algorithm is extended to solve the robust optimal control problem for linear continuoustime stochastic systems with input-dependent noise. Two examples are presented in Section 6. Finally, the conclusion is drawn in Section 7.

Notations: Throughout this paper, $I_{n}$ denotes the identity matrix of dimension $n$. $\mathbb{R}$ and $\mathbb{R}_{+}$denote the set of real numbers and the set of nonnegative real numbers, respectively. $\mathbb{Z}_{+}$denotes the set of nonnegative integers. $|\cdot|$ denotes the Euclidean norm for vectors, or the induced matrix norm for matrices. $\mathscr{S}^{n}$ denotes the normed space of all $n$-by- $n$ real symmetric matrices, equipped with the induced matrix norm. $\mathscr{S}_{+}^{n}=\{P \in$ $\left.\mathscr{S}^{n}: P \geq 0\right\}$. For a matrix $A \in \mathbb{R}^{n \times m}, A^{\dagger}$ denotes the pseudoinverse of $A ; \operatorname{vec}(A)=\left[a_{1}^{T}, a_{2}^{T}, \cdots, a_{m}^{T}\right]^{T}$, where $a_{i} \in \mathbb{R}^{n}$ is the $i$-th column of $A$. For any $A \in \mathscr{S}^{n}$, denote $\operatorname{vecs}(A)=\left[a_{11}, a_{12}, \cdots, a_{1 n}, a_{22}, a_{23}, \cdots, a_{n-1 n}, a_{n n}\right]^{T}$, where $a_{i j} \in \mathbb{R}$ is the $(i, j)$-th element of matrix $A$. $\otimes$ indicates the Kronecker product. A function $f: Q \rightarrow \mathbb{R}_{+}$, where $Q \subseteq \mathbb{R}^{n}$ and $0 \in Q$, is called positive definite, if $f(x)>0$ for all $x \in Q \backslash\{0\}$, and $f(0)=0$. A function $f$ is of class $\mathscr{C}^{0}(Q)$, where $Q \subseteq \mathbb{R}^{n}$, if $f$ is continuous on $Q$. For a continuously differentiable function $V: \mathbb{R}^{n} \rightarrow \mathbb{R}$, $\nabla V(x) \in \mathbb{R}^{1 \times n}$ denotes the gradient of $V$ at $x$. For any $0<T<\infty, \mathscr{D}\left([0, T], \mathscr{S}^{n}\right)$ denotes the space of functions from $[0, T]$ to $\mathscr{S}^{n}$, that are right-continuous with left-hand limits, equipped with the Skorokhod topology (Skorokhod, 1956). 


\section{Mathematical Preliminaries}

\subsection{Review of stochastic approximation}

Stochastic approximation, also known as stochastic gradient descent in some literature, serves as an important tool to solve stochastic optimization problems. Over the past several decades, various stochastic approximation methods have been developed (Robbins and Monro, 1951; Ljung, 1977; Chen and Zhu, 1986; Borkar, 1998; Chen, 2002; Abounadi et al., 2002; Kushner and Yin, 2003; Andrieu et al., 2005). Consider the following stochastic approximation algorithm:

$$
\theta_{k+1}=\theta_{k}+\epsilon_{k} g\left(\theta_{k}, w_{k}\right)+Z_{k}
$$

where $g$ is a nonlinear function, $\left\{w_{k}\right\}_{k=1}^{\infty}$ is a sequence of i.i.d. zero-mean random noise, $\epsilon_{k}$ is the step size, and $Z_{k}$ is the projection term. Assume

$$
\epsilon_{k}>0, \quad \sum_{k=0}^{\infty} \epsilon_{k}=\infty, \quad \sum_{k=0}^{\infty} \epsilon_{k}^{2}<\infty
$$

and the following dynamical system

$$
\dot{\theta}=\mathcal{E}_{w} g(\theta, w)
$$

where $\mathcal{E}$ represents the expectation operator, is asymptotically stable at a fixed-point $\theta^{*}$, then under some mild conditions on $\theta_{0}, w_{k}$ and $Z_{k}$, one can show (Kushner and Yin, 2003) that $\lim _{k \rightarrow \infty} \theta_{k}=\theta^{*}$ with probability one.

In this paper, we develop a continuous-time VI algorithm for linear systems based on the stochastic approximation theory.

\subsection{System description}

This paper considers the following linear system:

$$
\dot{x}=A x+B u, \quad x(0)=\xi,
$$

where $x \in \mathbb{R}^{n}$ is the system state, $u \in \mathbb{R}^{m}$ is the input, and $A \in \mathbb{R}^{n \times n}$ and $B \in \mathbb{R}^{n \times m}$ are unknown matrices. Assume $(A, B)$ is stabilizable.

Denote the cost corresponding to (2) as

$$
\mathcal{J}(\xi ; u)=\int_{0}^{\infty}\left(x^{T} Q x+u^{T} R u\right) d s, \quad x(0)=\xi
$$

where $Q=Q^{T} \geq 0, R=R^{T}>0$, and $\left(A, Q^{1 / 2}\right)$ is observable. It is well known that $\mathcal{J}$ is minimized under the optimal controller $u^{*}=-K^{*} x$, where $K^{*}=R^{-1} B^{T} P^{*}$, with $P^{*} \in \mathscr{S}^{n}$ the unique positive definite solution to the following ARE:

$$
A^{T} P+P A-P B R^{-1} B^{T} P+Q=0 .
$$

Since (3) is nonlinear in $P$, it is usually difficult to obtain the analytical solution directly. In this paper, a new method is proposed to iteratively approximate $P^{*}$.

\subsection{DMRE and DP mapping}

For any $M \in \mathscr{S}_{+}^{n}, \xi \in \mathbb{R}^{n}, t_{0} \in \mathbb{R}$, and $\tau \geq 0$, define the continuous-time DP mapping on $\left[t_{0}, t_{0}+\tau\right]$ as

$$
\begin{aligned}
\mathcal{T}_{\left[t_{0}, t_{0}+\tau\right]}(M, \xi):= & \min _{u \in \mathscr{C}^{0}\left(\left[t_{0}, t_{0}+\tau\right]\right)}\left\{x^{T}\left(t_{0}+\tau\right) M x\left(t_{0}+\tau\right)\right. \\
& \left.+\int_{t_{0}}^{t_{0}+\tau}\left(x^{T}(s) Q x(s)+u^{T}(s) R u(s)\right) d s\right\},
\end{aligned}
$$

where $x(\cdot)$ is the solution to (2) with the initial value $x\left(t_{0}\right)=\xi$. Note that $\mathcal{T}_{\left[t_{0}, t_{0}+\tau\right]}(\cdot, \cdot)$ is well established, and the finite-horizon minimization problem is solved with $u(s)=-R^{-1} B^{T} P(s) x(s)$, where $s \in\left[t_{0}, t_{0}+\tau\right]$, and $P(\cdot)$ is the solution to the following DMRE:

$$
-\dot{P}=A^{T} P+P A-P B R^{-1} B^{T} P+Q,
$$

with $P\left(t_{0}+\tau\right)=M$. Moreover, $\mathcal{T}_{\left[t_{0}, t_{0}+\tau\right]}(M, \xi)=$ $\xi^{T} P\left(t_{0}\right) \xi$. See, e.g., Kučera (1973) for more details.

Remark 2.1 It is not difficult to see that the definition of $\mathcal{T}_{\left[t_{0}, t_{0}+\tau\right]}(\cdot, \cdot)$ matches the definition of DP mapping for MDPs in Bertsekas (200\%, eq. (1.4)). Moreover, since (4) is time invariant, $\mathcal{T}_{\left[t_{0}, t_{0}+\tau\right]}(\cdot, \cdot)$ is independent of $t_{0}$, and thus $\mathcal{T}_{\left[t_{0}, t_{0}+\tau\right]}(\cdot, \cdot)=\mathcal{T}_{[0, \tau]}(\cdot, \cdot)$. For simplicity, we denote $\mathcal{T}_{\tau}(\cdot, \cdot):=\mathcal{T}_{[0, \tau]}(\cdot, \cdot)$ in the rest of this section.

We first state a technical lemma.

Lemma 2.2 Given $M_{0} \in \mathscr{S}_{+}^{n}$ and $\tau>0$, define $\left\{M_{k}\right\}_{k=1}^{\infty}$ as follows:

$$
\xi^{T} M_{k} \xi=\mathcal{T}_{\tau}\left(M_{k-1}, \xi\right), \quad \forall \xi \in \mathbb{R}^{n}
$$

Then, $\lim _{k \rightarrow \infty} M_{k}=P^{*}$, and

$$
\xi^{T} P^{*} \xi=\mathcal{T}_{t}\left(P^{*}, \xi\right), \quad \forall t>0, \forall \xi \in \mathbb{R}^{n} .
$$

Moreover, if $M_{N}>0$ for some integer $N \geq 0$, then $M_{k}>0, \forall k \geq N$.

PROOF. Letting $P(0)=M_{0}$, we can solve (4) on the time interval $[-\tau, 0]$. By $(5)$ and the definition of $\mathcal{T}_{\tau}(\cdot, \cdot)$, 
$P(-\tau)=M_{1}$. Similarly, at step $k$, solving (4) on the time interval $[-(k+1) \tau,-k \tau]$ with the boundary condition $P(-k \tau)=M_{k}$, we easily have $P(-(k+1) \tau)=M_{k+1}$. Thus, from mathematical induction, one can construct a sequence $\{P(-k \tau)\}_{k=0}^{\infty}$ from the solution to eq. (4), with $P(-k \tau)=M_{k}$.

Since $P(0)=M_{0} \in \mathscr{S}_{+}^{n}$, we have from Kučera (1973, Theorem 17) that,

$$
\lim _{k \rightarrow \infty} M_{k}=\lim _{t \rightarrow-\infty} P(t)=P^{*} .
$$

Moreover, (6) follows directly from (3) and (5).

By the definition of $\mathcal{T}_{\left[t_{0}, t_{0}+\tau\right]}(\cdot, \cdot)$, for any $\xi \in \mathbb{R}^{n}, t_{0}=$ $-(k+1) \tau$,

$$
\begin{aligned}
& \xi^{T} P(-(k+1) \tau) \xi \\
= & x^{T}(-k \tau) P(-k \tau) x(-k \tau) \\
& +\int_{-(k+1) \tau}^{-k \tau} x^{T}(s)\left(Q+P(s) B R^{-1} B^{T} P(s)\right) x(s) d s,
\end{aligned}
$$

where $x(\cdot)$ is the solution to the following closed-loop system on the time interval $[-(k+1) \tau,-k \tau]$ :

$$
\dot{x}(t)=\left(A-B R^{-1} B^{T} P(t)\right) x(t), \quad x(-(k+1) \tau)=\xi,
$$

and $P(\cdot)$ is the solution to $(4)$. Denote $\Phi(\cdot, \cdot)$ as the transition matrix of the above linear system. Then, it follows from (7) that

$$
\begin{aligned}
& P(-(k+1) \tau) \\
= & \Phi^{T}(-k \tau,-(k+1) \tau) P(-k \tau) \Phi(-k \tau,-(k+1) \tau) \\
& +\int_{-(k+1) \tau}^{-k \tau} \Phi^{T}(s,-(k+1) \tau)\left(Q+P(s) B R^{-1} B^{T} P(s)\right) \\
& \times \Phi(s,-(k+1) \tau) d s .
\end{aligned}
$$

Since $\Phi(\cdot, \cdot)$ is nonsingular, by induction, if $M_{N}=$ $P(-N \tau)>0, M_{k}=P(-k \tau)>0$ for all $k \geq N$.

Remark 2.3 Note that (5) is similar to the VI algorithm for discrete-time linear systems (Bertsekas, 2005, Proposition 4.4.1) and MDPs (Puterman, 1994, Theorem 6.3.1). Indeed, if one applies $\mathcal{T}_{\tau}(\cdot, \cdot)$ to discrete-time linear systems, and only takes one time step $(\tau=1)$, then

$$
\begin{aligned}
& \mathcal{T}_{1}(M, \xi) \\
= & \min _{u(0) \in \mathbb{R}^{m}}\left\{x^{T}(1) M x(1)+\xi^{T} Q \xi+u^{T}(0) R u(0)\right\} \\
= & \min _{v \in \mathbb{R}^{m}}\left\{(A \xi+B v)^{T} M(A \xi+B v)+\xi^{T} Q \xi+v^{T} R v\right\} \\
= & \xi^{T}\left(A^{T} M A+Q-A^{T} M B\left(R+B^{T} M B\right)^{-1} B^{T} M A\right) \xi,
\end{aligned}
$$

and (5) is exactly the discrete-time VI algorithm in Bertsekas (2005, Proposition 4.4.1).
Following the proof of Lemma 2.2, we reverse the timeline in (4) to obtain

$$
\dot{P}=A^{T} P+P A-P B R^{-1} B^{T} P+Q, P(0) \in \mathscr{S}_{+}^{n} .
$$

Obviously, $\lim _{t \rightarrow \infty} P(t)=P^{*}$. Also, if $P(\tau)>0$ for some $\tau \geq 0$, then $P(t)>0$ for all $t \geq \tau$.

Although the convergence of (5) and (8) is guaranteed, it is not easy to solve the analytic solution directly. In the next section, we develop an off-line iterative algorithm to approximate $P^{*}$. The online ADP methods based on this algorithm are presented in Sections 4 and 5 .

\section{Iterative scheme for continuous-time VI}

In this section, a continuous-time VI algorithm is derived. First, we give several definitions. $\left\{B_{q}\right\}_{q=0}^{\infty}$ is a collection of bounded sets with nonempty interiors, and satisfying

$$
B_{q} \subseteq B_{q+1}, \quad q \in \mathbb{Z}_{+}, \quad \lim _{q \rightarrow \infty} B_{q}=\mathscr{S}_{+}^{n}
$$

Let $\bar{\varepsilon}>0$ be a small threshold.

The continuous-time VI algorithm is described in details in Algorithm 1.

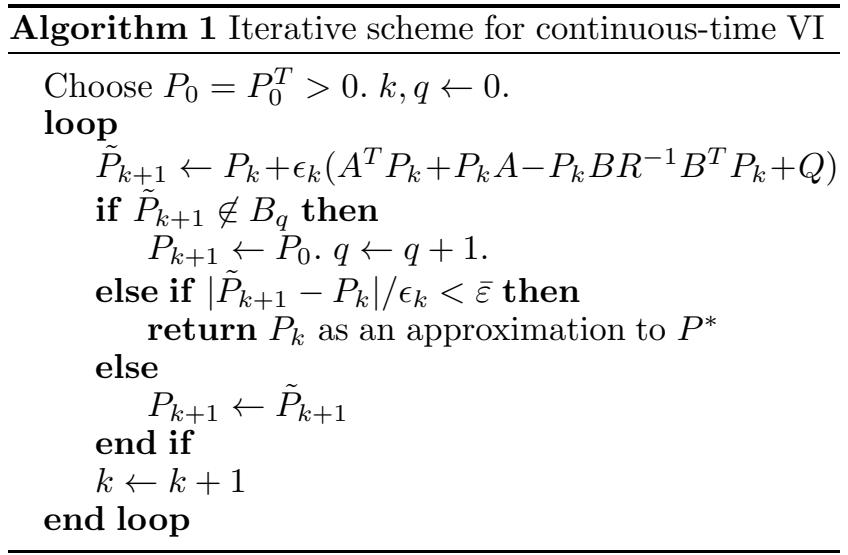

Remark 3.1 Algorithm 1 can be easily extended to more general forms. For example, $\left\{\epsilon_{k}\right\}_{k=0}^{\infty}$ may be a sequence of random variables. In (1), $\sum_{k=0}^{\infty} \epsilon_{k}^{2}<\infty$ can be replaced by $\lim _{k \rightarrow \infty} \epsilon_{k}=0$, when $\left\{\epsilon_{k}\right\}_{k=0}^{\infty}$ is a deterministic sequence. Also, when $\tilde{P}_{k+1} \notin B_{q}$, it is sufficient to select $P_{k+1}$ from a fixed bounded subset of the interior of $\mathscr{S}_{+}^{n}$. Notice that when Algorithm 1 is implemented in practice, according to Cauchy criterion, the stopping criterion $\left|\tilde{P}_{k+1}-P_{k}\right| / \epsilon_{k}<\bar{\varepsilon}$ should be verified for several consecutive values of $k$. For simplicity, we do not consider these more general conditions in this paper. 
Remark 3.2 Compared with PI, i.e., Kleinman's algorithm (Kleinman, 1968), Algorithm 1 has two advantages. First, a stabilizing control gain matrix is not required to start the iteration. Second, there is no need to solve a matrix equation (i.e., the Lyapunov equation) at each iteration.

The convergence result of Algorithm 1 is given in the following theorem.

Theorem 3.3 Given $\left\{P_{k}\right\}_{k=0}^{\infty}$ from Algorithm 1, $\lim _{k \rightarrow \infty} P_{k}=P^{*}$.

Before giving the proof for Theorem 3.3, two lemmas are given.

The following result shows the boundedness of $\left\{P_{k}\right\}_{k=0}^{\infty}$. The proof of Lemma 3.4 is inspired by the proof of Andrieu et al. (2005, Lemma 2.1), and has been postponed to Appendix A.

Lemma 3.4 Given $\left\{P_{k}\right\}_{k=0}^{\infty}$ defined in Algorithm 1, there exist $N \geq 0$ and a compact set $\mathcal{K} \subset \mathscr{S}_{+}^{n}$ with nonempty interior, such that $\left\{P_{k}\right\}_{k=N}^{\infty} \subset \mathcal{K}$, and $P^{*} \in \mathcal{K}$.

Next, an extended version of Arzelá-Ascoli theorem (Billingsley, 2009, Theorem 12.3) is introduced.

Lemma 3.5 $A$ set $A \subset \mathscr{D}\left([0, T], \mathscr{S}^{n}\right)$ is relatively compact, if and only if

$$
\sup _{x \in A} \sup _{t \in[0, T]}|x(t)|<\infty
$$

and, for any $\varepsilon>0$, there exists $\delta>0$, such that

$$
\sup _{x \in A} \omega_{x}^{\prime}(\delta)<\varepsilon,
$$

where

$$
\omega_{x}^{\prime}(\delta)=\inf _{\left\{\tau_{i}\right\}_{i=0}^{v}} \max _{1 \leq i \leq v} \sup _{t, s \in\left[\tau_{i-1}, \tau_{i}\right)}|x(t)-x(s)|,
$$

and $\left\{\tau_{i}\right\}_{i=0}^{v}$ is a partition of the interval $[0, T]$ with $\min _{1 \leq i \leq v}\left(\tau_{i}-\tau_{i-1}\right)>\delta$.

Remark 3.6 The second condition in Lemma 3.5 holds if for all $x \in A$, any two discontinuity points in $[0, T]$ are separated by at least some $\Delta>0$. In this case, $\max _{1 \leq i \leq v} \sup _{t, s \in\left[\tau_{i-1}, \tau_{i}\right)}|x(t)-x(s)|$ can be made arbitrarily small by choosing a small enough $\delta>0$, and assigning each discontinuity point a corresponding $\tau_{i}$.

Now, we are ready to give the proof of Theorem 3.3.

Proof of Theorem 3.3. The proof is similar to the methods in Kushner and Yin (2003, Chapter 5) and
Abounadi et al. (2002). First, based on Lemma 3.4 and the definition of $\mathcal{K}$, one has from Algorithm 1 that

$$
\begin{aligned}
P_{k+1}=P_{k} & +\epsilon_{k}\left(A^{T} P_{k}+P_{k} A-P_{k} B R^{-1} B^{T} P_{k}+Q\right) \\
& +Z_{k}, \quad \forall k \geq N,
\end{aligned}
$$

and $P_{k} \in \mathcal{K}$, where the projection term $Z_{k}$ is defined as

$$
Z_{k}= \begin{cases}P_{0}-\tilde{P}_{k+1}, & \text { if } \tilde{P}_{k+1} \notin \mathcal{K} \\ 0, & \text { otherwise }\end{cases}
$$

Consider the following continuous-time interpolation:

$$
P^{0}(t)= \begin{cases}P_{0}, & t \leq 0 \\ P_{k}, & t \in\left[t_{k}, t_{k+1}\right),\end{cases}
$$

where $t_{0}=0$ and $t_{k}=\sum_{i=0}^{k-1} \epsilon_{i}$, for $k \geq 1$. Define the shifted process $P^{k}(t)=P^{0}\left(t_{k}+t\right), \forall t \in(-\infty, \infty)$.

Then, we have for all $k \geq N$ and $t \geq 0$ that

$$
\begin{aligned}
P^{k}(t)= & P_{k}+\sum_{i=k}^{m\left(t+t_{k}\right)-1} \epsilon_{i}\left(A^{T} P_{i}+P_{i} A-P_{i} B R^{-1} B^{T} P_{i}+Q\right) \\
& +\sum_{i=k}^{m\left(t+t_{k}\right)-1} Z_{i} \\
= & P_{k}+H^{k}(t)+Z^{k}(t)+e^{k}(t),
\end{aligned}
$$

where

$$
\begin{aligned}
& H^{k}(t)=\int_{0}^{t}\left(A^{T} P^{k}(s)+P^{k}(s) A-P^{k}(s) B R^{-1} B^{T} P^{k}(s)+Q\right) d s, \\
& Z^{k}(t)=\sum_{i=k}^{m\left(t+t_{k}\right)-1} Z_{i}, \quad m(t)= \begin{cases}j, & 0 \leq t_{j} \leq t<t_{j+1}, \\
0, & t<0,\end{cases}
\end{aligned}
$$

$e^{k}(t)$ is due to replacing the second term on the righthand side of the first equality in (9) with $H^{k}(t)$, and $N$ is chosen as in Lemma 3.4. By convention, the above definition assumes $\sum_{i=k}^{m\left(t+t_{k}\right)-1} *=0$, when $0 \leq t<\epsilon_{k}$.

Fixing $T>0$, we next show that $\left\{H^{k}(\cdot)\right\}_{k=N}^{\infty}$, $\left\{Z^{k}(\cdot)\right\}_{k=N}^{\infty}$, and $\left\{e^{k}(\cdot)\right\}_{k=N}^{\infty}$ are relatively compact in $\mathscr{D}\left([0, T], \mathscr{S}^{n}\right)$.

By Lemma 3.4, $H^{k}(\cdot)$ is uniformly bounded on $[0, \infty)$. Then, since $H^{k}(\cdot) \in \mathscr{C}^{0}\left(\mathbb{R}_{+}\right), \forall k \geq 0$, we know by Lemma 3.5 that $\left\{H^{k}(\cdot)\right\}_{k=N}^{\infty}$ is relatively compact in $\mathscr{D}\left([0, T], \mathscr{S}^{n}\right)$.

Now consider $\left\{e^{k}(\cdot)\right\}_{k=N}^{\infty}$. Note that $e^{k}(\cdot)$ is continuous 
except at $t=t_{j}-t_{k}, \forall j>k$, and

$$
\begin{aligned}
e^{k}(t)=0, \quad \text { if } t=t_{j}-t_{k}, \exists j \geq k ; & \\
\left|e^{k}(t)\right|< & \epsilon_{j} \mid A^{T} P^{0}\left(t_{j}\right)+P^{0}\left(t_{j}\right) A-P^{0}\left(t_{j}\right) B R^{-1} B^{T} P^{0}\left(t_{j}\right) \\
& +Q \mid, \quad \text { if } t_{j}-t_{k}<t<t_{j+1}-t_{k}, \exists j \geq k .
\end{aligned}
$$

Since $\left\{P_{k}\right\}_{k=0}^{\infty} \subset \mathcal{K}, \lim _{k \rightarrow \infty} e^{k}(\cdot)=0$ uniformly on $[0, \infty)$. Thus, for any $\varepsilon>0$, one can find $k^{\prime} \geq N$, such that for all $k>k^{\prime}, \sup _{0 \leq t \leq T}\left|e^{k}(t)\right|<\varepsilon$. By definition, for all $k \in\left[N, k^{\prime}\right], e^{k}(\cdot)$ has a finite number of discontinuity points in $[0, T]$. Then, there exists $\delta>0$, such that for any $e^{k}(\cdot) \in\left\{e^{k}(\cdot)\right\}_{k=N}^{k^{\prime}}$, any two discontinuity points of $e^{k}(\cdot)$ in $[0, T]$ are separated by at least $\delta$. Thus, by Lemma $3.5,\left\{e^{k}(\cdot)\right\}_{k=N}^{\infty}$ is relatively compact in $\mathscr{D}\left([0, T], \mathscr{S}^{n}\right)$.

Next, to show $\left\{Z^{k}(\cdot)\right\}_{k=N}^{\infty}$ is relatively compact in $\mathscr{D}\left([0, T], \mathscr{S}^{n}\right)$, note that since $\left\{P_{k}\right\}_{k=N}^{\infty} \subset \mathcal{K}$, there exist $k^{\prime} \geq N$ and $T^{\prime} \in\left(\epsilon_{k^{\prime}}, T\right]$, such that for all $k \geq k^{\prime}$, $\epsilon_{k} \leq \epsilon_{k^{\prime}}$, and if $P_{k}=P_{0}$, then $P^{k}\left(0^{-}\right) \in \partial \mathcal{K}$ and

$$
P_{k}+H^{k}(t)+e^{k}(t) \in \mathcal{K}, \quad \forall t \in\left[0, T^{\prime}\right] .
$$

Therefore, $Z_{j}=0, \forall j \in\left[k, m\left(t+t_{k}\right)-1\right], \forall t \in\left[\epsilon_{k}, T^{\prime}\right]$, and thus $Z^{k}(\cdot) \equiv 0$ on $\left[0, T^{\prime}\right], \forall k \geq k^{\prime}$. This implies that for all $k \geq k^{\prime}$ with $P_{k}=P_{0}$, any two discontinuity points of $Z^{k}(\cdot)$ are separated by at least $T^{\prime}$. For $k \in\left[N, k^{\prime}\right)$, since $Z^{k}(\cdot)$ is piecewise constant on $[0, T]$, we easily know that $Z^{k}(\cdot)$ has a finite number of discontinuity points in $[0, T]$. Thus, $\left\{Z^{k}(\cdot)\right\}_{k=N}^{\infty}$ is relatively compact in $\mathscr{D}\left([0, T], \mathscr{S}^{n}\right)$.

Since $\left\{H^{k}(\cdot)\right\}_{k=N}^{\infty},\left\{Z^{k}(\cdot)\right\}_{k=N}^{\infty}$, and $\left\{e^{k}(\cdot)\right\}_{k=N}^{\infty}$ are relatively compact in $\mathscr{D}\left([0, T], \mathscr{S}^{n}\right), \forall T>0$, by definition, $\left\{P^{k}(\cdot)\right\}_{k=N}^{\infty}$ is also relatively compact in $\mathscr{D}\left([0, T], \mathscr{S}^{n}\right)$, $\forall T>0$. Then (Billingsley, 2009, pp. 240), for a converging subsequence $\left\{\left(P^{k^{\prime}}(\cdot), Z^{k^{\prime}}(\cdot)\right)\right\}$, the limit $(P(\cdot), Z(\cdot))$ satisfies

$$
\begin{aligned}
P(t)=P(0) & +\int_{0}^{t}\left(A^{T} P(s)+P(s) A\right. \\
& \left.-P(s) B R^{-1} B^{T} P(s)+Q\right) d s+Z(t)
\end{aligned}
$$

for all $t \geq 0$. Following the procedure in the proof of Abounadi et al. (2002, Lemma 3.4), one can easily show that if $Z(\cdot) \not \equiv 0$, then there exists $\tau>0$, such that $P\left(\tau^{-}\right) \in \partial \mathcal{K}$ and $P\left(\tau^{+}\right)=P_{0}$. However, by the definition of $\mathcal{K}$ in Lemma 3.4 and the asymptotic stability property of system (8), this is impossible. Thus, the limit $P(\cdot)$ satisfies

$$
\dot{P}=A^{T} P+P A-P B R^{-1} B^{T} P+Q .
$$

Since $P^{*} \in \mathcal{K} \subset R_{A},\left\{P_{k}\right\}_{k=N}^{\infty}$ remains in the region of attraction of $P^{*}$. By Kushner and Yin (2003, Theorem 5.2.1), $\lim _{k \rightarrow \infty} P_{k}=P^{*}$. This completes the proof.
Remark 3.7 Due to Lemma 3.4, Theorem 3.3 appears as a deterministic analogue of Kushner and Yin (2003, Theorem 5.2.1). However, the projection term $Z_{k}$ defined in this paper is slightly different from the projection term in Kushner and Yin (2003, eq. (5.1.4)).

Remark 3.8 If the upper-bound of $\left|P^{*}\right|$ is known a priori, we can simply fix $B_{q} \equiv \bar{B}$ for all $q \in \mathbb{Z}_{+}$, where $\bar{B}$ is bounded, with $P_{0}$ and $P^{*}$ in its interior. Then, Lemma 3.4 holds with $N=0$, and the proof of Theorem 3.3 remains almost unchanged. In this case, the convergence of the VI algorithm is still guaranteed.

Thanks to the convergence result in Theorem 3.3, using a stopping criterion, we obtain a matrix, say $\hat{P}^{*}$, from Algorithm 1, and the corresponding suboptimal feedback gain matrix $\hat{K}^{*}=R^{-1} B^{T} \hat{P}^{*}$. However, it is important to notice that system (2) under $u=-\hat{K}^{*} x$ may not be globally asymptotically stable (GAS) at the origin. The following corollary provides additional conditions on $Q$ and $\bar{\varepsilon}$ under which such a control law is stabilizing.

Corollary 3.9 Assume $Q>\bar{\varepsilon} I_{n}$. Then, from Algorithm 1 we can find a stabilizing control law of the form $u=$ $-\hat{K}^{*} x$ for system (2). That is, $A-B \hat{K}^{*}$ is Hurwitz.

PROOF. By the definition,

$$
A^{T} \hat{P}^{*}+\hat{P}^{*} A-\hat{P}^{*} B R^{-1} B^{T} \hat{P}^{*}+Q<\bar{\varepsilon} I_{n} .
$$

Since $Q>\bar{\varepsilon} I_{n}$,

$$
\begin{gathered}
\left(A-B \hat{K}^{*}\right)^{T} \hat{P}^{*}+\hat{P}^{*}\left(A-B \hat{K}^{*}\right) \\
<-Q+\bar{\varepsilon} I_{n}-\left(\hat{K}^{*}\right)^{T} R \hat{K}^{*}<0 .
\end{gathered}
$$

Therefore, $A-B \hat{K}^{*}$ is Hurwitz.

Similar to the discrete-time VI algorithm, Algorithm 1 can also be applied to solve the policy evaluation in Kleinman's algorithm.

Corollary 3.10 Given any $K \in \mathbb{R}^{n \times m}$ such that $A-$ $B K$ is Hurwitz, we apply Algorithm 1 with the updating equation of $\tilde{P}_{k+1}$ replaced by

$$
\begin{aligned}
\tilde{P}_{k+1} \leftarrow P_{k} & +\epsilon_{k}\left((A-B K)^{T} P_{k}+P_{k}(A-B K)\right. \\
& \left.+Q+K^{T} R K\right) .
\end{aligned}
$$

Then, $\lim _{k \rightarrow \infty} P_{k}=P$, where $P$ is the solution to

$$
(A-B K)^{T} P+P(A-B K)+Q+K^{T} R K=0 .
$$


PROOF. Since $A-B K$ is Hurwitz, by the definition of $P$, for any $t_{0} \in[0, \infty)$,

$$
\begin{aligned}
& x^{T}\left(t_{0}\right) P x\left(t_{0}\right) \\
= & \lim _{t \rightarrow \infty} x^{T}(t) P x(t)+\int_{t_{0}}^{\infty} x^{T}(s)\left(Q+K^{T} R K\right) x(s) d s \\
= & \int_{t_{0}}^{\infty} x^{T}(s)\left(Q+K^{T} R K\right) x(s) d s,
\end{aligned}
$$

where $x(\cdot)$ is the solution to the following closed-loop system:

$$
\dot{x}=(A-B K) x .
$$

Thus, $P=P^{T} \geq P^{*}>0$, and

$$
0<\int_{t_{0}}^{\infty} \Phi^{T}\left(s, t_{0}\right)\left(Q+K^{T} R K\right) \Phi\left(s, t_{0}\right) d s<\infty
$$

where $\Phi(\cdot, \cdot)$ is the transition matrix of system (10). By continuity, there exists $T \in\left(t_{0}, \infty\right)$, such that

$$
\int_{t_{0}}^{T} \Phi^{T}\left(s, t_{0}\right)\left(Q+K^{T} R K\right) \Phi\left(s, t_{0}\right) d s>0
$$

Thus, the pair $\left(A-B K, \sqrt{Q+K^{T} R K}\right)$ is observable. By definition, $P$ is the solution to the LQR problem composed with system (10) (where the input matrix is 0 ) and the following cost:

$$
\mathcal{J}(x(0) ; u)=\int_{0}^{\infty}\left(x^{T}\left(Q+K^{T} R K\right) x+u^{T} R u\right) d s .
$$

Then, we have by Theorem $3.3 \lim _{k \rightarrow \infty} P_{k}=P$. This completes the proof.

Since $\lim _{k \rightarrow \infty} P_{k}=P^{*}$, there exists $N<\infty$, such that $\tilde{P}_{k}=P_{k}$ for all $k \geq N$. Then, the updating equation in Algorithm 1 reduces to

$$
P_{k+1} \leftarrow P_{k}+\epsilon_{k}\left(A^{T} P_{k}+P_{k} A+Q-P_{k} B R^{-1} B^{T} P_{k}\right),
$$

for all $k \geq N$.

Corollary 3.11 The sequence $\left\{P_{k}\right\}_{k=N}^{\infty}$ converges sublinearly to $P^{*}$ in Euclidean norm.
PROOF. It can be directly checked that

$$
\begin{aligned}
& P^{*}-P_{k+1} \\
= & P^{*}-P_{k}+\epsilon_{k}\left(A^{T} P^{*}+P^{*} A+Q-P^{*} B R^{-1} B^{T} P^{*}\right) \\
& -\epsilon_{k}\left(A^{T} P_{k}+P_{k} A+Q-P_{k} B R^{-1} B^{T} P_{k}\right) \\
= & \left(\frac{1}{2} I_{n}+\epsilon_{k} A^{*}\right)^{T} \Delta P_{k}+\Delta P_{k}\left(\frac{1}{2} I_{n}+\epsilon_{k} A^{*}\right) \\
& +\epsilon_{k} \Delta P_{k} B R^{-1} B^{T} \Delta P_{k},
\end{aligned}
$$

where $A^{*}=A-B K^{*}$, and $\Delta P_{k}=P^{*}-P_{k}$.

Since $\lim _{k \rightarrow \infty} P_{k}=P^{*}, \lim _{k \rightarrow \infty} \epsilon_{k}=0$, and $\epsilon_{k}>0$ for all $k \in \mathbb{Z}_{+}$,

$$
\lim _{k \rightarrow \infty} \frac{\left|\Delta P_{k+1}\right|}{\left|\Delta P_{k}\right|} \leq \lim _{k \rightarrow \infty}\left\{\lambda_{k}+\epsilon_{k}\left|B R^{-1} B^{T}\right| \cdot\left|\Delta P_{k}\right|\right\}=1,
$$

where $\lambda_{k}=\left|I_{n}+2 \epsilon_{k} A^{*}\right|$. This completes the proof.

Remark 3.12 Due to the decreasing stepsize $\epsilon_{k}$, the tail process of $\left\{P_{k}\right\}_{k=0}^{\infty}$ in Algorithm 1 only converges sublinearly. This is not surprising, since many stochastic approximation-based methods, including Robbins-Monro algorithm (Robbins and Monro, 1951), also converge in sublinear rate. The order of the convergence rate depends on $A-B K^{*}$ and the choice of $\epsilon_{k}$. Moreover, $\epsilon_{k}$ and $\lambda_{k}$ play a similar role as the discounting factor in discounted Markov decision problems (Puterman, 1994, Theorem 6.3.3).

Remark 3.13 Note that we do not assume the DP mapping and the dynamics of DMRE satisfy any forms of contraction property in the proofs of Theorem 3.3 and Corollary 3.11. In fact, our analysis on the continuoustime VI algorithm can be regarded as an extension of the noncontractive model in abstract DP framework (Bertsekas, 2013, Chapter 4).

Before closing this section, it is worth noting that continuous VI was also studied in Vrabie et al. (2013, Chapter 6) without a convergence proof. Interestingly, as we will show below, Vrabie's algorithm is rather a modified PI algorithm (Puterman, 1994, Chapter 6.5).

For simplicity, denote

$$
\begin{array}{r}
H(P, K)=(A-B K)^{T} P+P(A-B K)+Q+K^{T} R K, \\
\forall P \in \mathscr{S}^{n}, K \in \mathbb{R}^{m \times n} .
\end{array}
$$

The following Lemma 3.14 is a continuous-time counterpart of Puterman (1994, Theorem 6.2.2).

Lemma 3.14 Given $P \in \mathscr{S}_{+}^{n}$, if $H(P, K) \leq 0$, where $K=R^{-1} B^{T} P$, then $P^{*} \leq P$. 
PROOF. Let $x(\cdot)$ be the solution to system (2) with $u=-K x$ and an arbitrary initial value $x(0) \in R^{n}$. Differentiating the Lyapunov function candidate $x^{T} P x$ along the solution yields

$$
\frac{d}{d s}\left(x^{T}(s) P x(s)\right)=2 x^{T}(s)(A-B K)^{T} P x(s) .
$$

Integrating the above equation over $[0, t]$, and using the Comparison Lemma (Khalil, 2002, Lemam 3.4) and the fact that $H(P, K) \leq 0$, it follows that

$$
\begin{aligned}
x^{T}(0) P x(0) & \geq x^{T}(t) P x(t)+\int_{0}^{t} x^{T}(s)\left(K^{T} R K+Q\right) x(s) d s \\
& \geq \int_{0}^{t} x^{T}(s)\left(K^{T} R K+Q\right) x(s) d s .
\end{aligned}
$$

Since the above inequality holds for all $t>0$, one has

$$
\begin{aligned}
x^{T}(0) P x(0) & \geq \int_{0}^{\infty} x^{T}(s)\left(K^{T} R K+Q\right) x(s) d s \\
& \geq x^{T}(0) P^{*} x(0) .
\end{aligned}
$$

Thus, $P^{*} \leq P$.

Next, we give the convergence result for Vrabie's VI algorithm.

\section{Proposition 3.15 Given $P_{0} \in \mathscr{S}^{n}$ satisfying}

$$
A^{T} P_{0}+P_{0} A-P_{0} B R^{-1} B^{T} P_{0}+Q \leq 0
$$

define $\left\{P_{k}\right\}_{k=1}^{\infty}$ as follows:

$$
P_{k+1}=e^{A_{k}^{T} t} P_{k} e^{A_{k} t}+\int_{0}^{t} e^{A_{k}^{T} s}\left(K_{k}^{T} R K_{k}+Q\right) e^{A_{k} s} d s
$$

where $k=0,1, \cdots, 0<t<\infty, K_{k}=R^{-1} B^{T} P_{k}$, and $A_{k}=A-B K_{k}$. Then,

- $P_{0} \geq P_{1} \geq \cdots \geq P^{*}$;

- $\lim _{k \rightarrow \infty} \bar{P}_{k}=\bar{P}^{*}, \lim _{k \rightarrow \infty} K_{k}=K^{*}$;

- $A_{k}$ is Hurwitz for all $k \geq 0$.

Remark 3.16 It can be seen from Proposition 3.15 that by letting $t$ go to infinity, $P_{k+1}$ in (12) approaches the solution to the policy evaluation step (Murray et al., 2002, eq. (12)). Thus, it is more appropriate to regard (12) as a modified PI (Puterman, 1994, Theorem 6.5.5) or an optimistic PI (Bertsekas, 2013, Proposition 4.4.3), rather than a VI algorithm. Indeed, (11) is similar to the conditions assumed in Bertsekas (2013, Proposition 4.4.3) and Puterman (1994, Theorem 6.5.5).
Proof of Proposition 3.15. From Lemma 3.14, $P_{0} \geq$ $P^{*}$. Moreover, using the proof techniques of Lemma 3.14, it can be easily shown that

$$
\begin{aligned}
P_{0} & \geq e^{A_{0}^{T} t} P_{0} e^{A_{0} t}+\int_{0}^{t} e^{A_{0}^{T} s}\left(Q+K_{0}^{T} R K_{0}\right) e^{A_{0} s} d s \\
& =P_{1} .
\end{aligned}
$$

Thus, $P_{1} \leq P_{0}$. This leads to

$$
\begin{aligned}
0 & \geq e^{A_{0}^{T} t} P_{1} e^{A_{0} t}-P_{1}+\int_{0}^{t} e^{A_{0}^{T} s}\left(K_{0}^{T} R K_{0}+Q\right) e^{A_{0} s} d s \\
& =\int_{0}^{t} e^{A_{0}^{T} s} H\left(P_{1}, K_{0}\right) e^{A_{0} s} d s \\
& =e^{A_{0}^{T} t^{\prime}} H\left(P_{1}, K_{0}\right) e^{A_{0} t^{\prime}} \geq e^{A_{0}^{T} t^{\prime}} H\left(P_{1}, K_{1}\right) e^{A_{0} t^{\prime}} .
\end{aligned}
$$

where $0 \leq t^{\prime} \leq t$, and the last equality follows from the mean value theorem for integrals. Thus, $H\left(P_{1}, K_{1}\right) \leq 0$. From Lemma 3.14, $P_{1} \geq P^{*}$. By induction, we have $P_{0} \geq P_{1} \geq \cdots \geq P^{*}$. Since $\left\{P_{k}\right\}_{k=0}^{\infty}$ is monotonically decreasing and is bounded from below by $P^{*}$, we know from monotone sequence theorem (Kantorovich and Akilov, 1982, pp. 169) that $\left\{P_{k}\right\}_{k=0}^{\infty}$ converges. Since $K_{k}$ is continuous in $P_{k},\left\{K_{k}\right\}_{k=0}^{\infty}$ also converges. Moreover, since $H\left(P_{k}, K_{k}\right) \leq 0$, by the observability assumption and LaSalle's invariance principle (Khalil, 2002, Theorem 4.4), $A_{k}$ is Hurwitz.

To show $\lim _{k \rightarrow \infty} P_{k}=P^{*}$, suppose $\lim _{k \rightarrow \infty} P_{k}=$ $\bar{P} \neq P^{*}$. Then, there exists $E=E^{T} \neq 0$, such that $H(\bar{P}, \bar{K})=E$, and thus

$$
\begin{aligned}
\bar{P}= & e^{A_{c}^{T} t} \bar{P} e^{A_{c} t}+\int_{0}^{t} e^{A_{c}^{T} s}\left(\bar{K}^{T} R \bar{K}+Q\right) e^{A_{c} s} d s \\
& -\int_{0}^{t} e^{A_{c}^{T} s} E e^{A_{c} s} d s,
\end{aligned}
$$

where $\bar{K}=R^{-1} B^{T} \bar{P}$, and $A_{c}=A-B \bar{K}$. Since $\lim _{k \rightarrow \infty} P_{k}=\bar{P}$, one has from (12) and (13) that (Rudin, 1987, Theorem 1.34)

$$
\int_{0}^{t} e^{A_{c}^{T} s} E e^{A_{c} s} d s=0
$$

Then, $E=0$, which contradicts to the definition of $E$. Thus, $\bar{P}=P^{*}$ and $\bar{K}=K^{*}$. This completes the proof.

\section{VI-based ADP design for linear continuous- time systems}

In this section, an on-line ADP algorithm for linear continuous- time systems is given based on Algorithm 1 . 
For all $x \in \mathbb{R}^{n}$, taking the derivative along the solutions of system (2), one has

$$
\begin{aligned}
\frac{d}{d t}\left(x^{T} P_{k} x\right) & =(A x+B u)^{T} P_{k} x+x^{T} P_{k}(A x+B u) \\
& =x^{T} H_{k} x+2(R u)^{T} K_{k} x,
\end{aligned}
$$

where $H_{k}=A^{T} P_{k}+P_{k} A$, and $K_{k}=R^{-1} B^{T} P_{k}$.

Integrating both sides of the above equation along the trajectories of (2), we have

$$
\begin{aligned}
\left.x^{T} P_{k} x\right|_{t} ^{t+\delta t}= & \int_{t}^{t+\delta t} x^{T} \otimes x^{T} d s \operatorname{vec}\left(H_{k}\right) \\
& +2 \int_{t}^{t+\delta t} x^{T} \otimes(R u)^{T} d s \operatorname{vec}\left(K_{k}\right),
\end{aligned}
$$

where $\delta t>0$. For any $\xi \in \mathbb{R}^{q}$, where $q$ is a positive integer, denote

$$
\bar{\xi}=\left[\xi_{1}^{2}, 2 \xi_{1} \xi_{2}, \cdots, 2 \xi_{1} \xi_{q}, \xi_{2}^{2}, 2 \xi_{2} \xi_{3}, \cdots, 2 \xi_{q-1} \xi_{q}, \xi_{q}^{2}\right] .
$$

Next, define three matrices:

$$
\begin{aligned}
\delta_{x x} & =\left[\left.x \otimes x\right|_{t_{0}} ^{t_{1}},\left.x \otimes x\right|_{t_{1}} ^{t_{2}}, \cdots,\left.x \otimes x\right|_{t_{l-1}} ^{t_{l}}\right]^{T}, \\
I_{x u} & =\left[\int_{t_{0}}^{t_{1}} x \otimes R u d s, \int_{t_{1}}^{t_{2}} x \otimes R u d s, \cdots, \int_{t_{l-1}}^{t_{l}} x \otimes R u d s\right]^{T}, \\
I_{x x} & =\left[\int_{t_{0}}^{t_{1}} \bar{x} d s, \int_{t_{1}}^{t_{2}} \bar{x} d s, \cdots, \int_{t_{l-1}}^{t_{l}} \bar{x} d s\right]^{T},
\end{aligned}
$$

where $l$ is a positive integer, and $0 \leq t_{0}<t_{1}<\cdots<$ $t_{l-1}<t_{l}$ are known constants.

Using the above notations, (15) can be rewritten in a more compact form:

$$
\left[I_{x x}, 2 I_{x u}\right]\left[\begin{array}{c}
\operatorname{vecs}\left(H_{k}\right) \\
\operatorname{vec}\left(K_{k}\right)
\end{array}\right]=\delta_{x x} \operatorname{vec}\left(P_{k}\right) .
$$

If $u$ is chosen to satisfy the following condition

$$
\operatorname{rank}\left(\left[I_{x x}, 2 I_{x u}\right]\right)=\frac{n(n+1)}{2}+n m,
$$

then (17) has unique solutions of $H_{k}$ and $K_{k}$, and one can easily solve

$$
\left[\begin{array}{c}
\operatorname{vecs}\left(H_{k}\right) \\
\operatorname{vec}\left(K_{k}\right)
\end{array}\right]=\Theta \operatorname{vec}\left(P_{k}\right)
$$

where $\Theta=\left[I_{x x}, 2 I_{x u}\right]^{\dagger} \delta_{x x}$.

Borrowing the terminology from traditional adaptive control (Tao, 2014; Åström and Wittenmark, 1997), such an input, usually chosen as sinusoidal signals or random noise, satisfying (18) is called "persistently existing".

Based on the above analysis, the ADP algorithm based on Algorithm 1 for linear continuous-time systems is given in Algorithm 2.

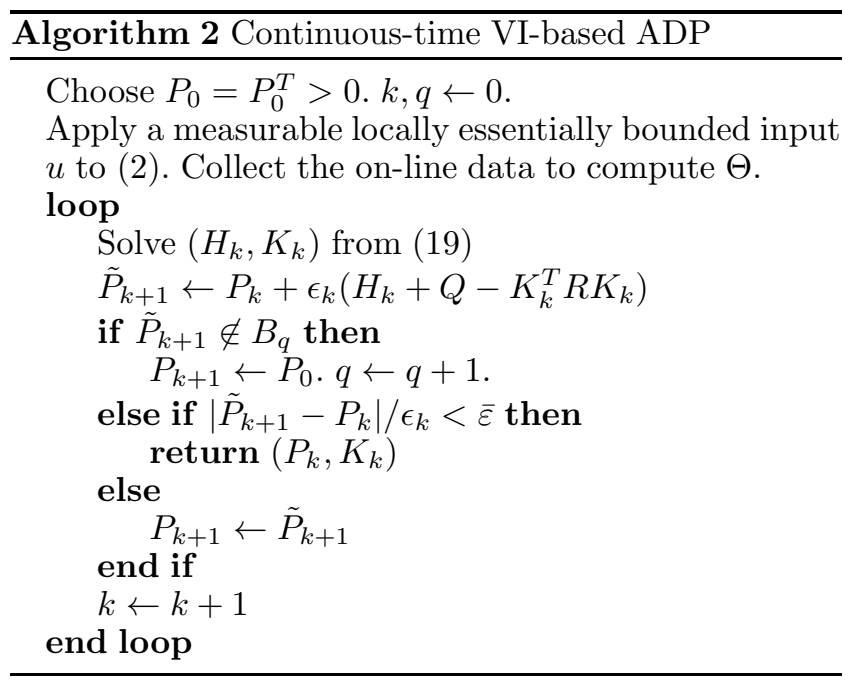

Remark 4.1 Note that Algorithm 2 is an on-line, offpolicy algorithm that does not require an initial stabilizing control policy and the knowledge of system matrices. Moreover, different from Jiang and Jiang (2012, eq. (11)), $K_{k}$ does not appear in the design matrix $\left[I_{x x}, 2 I_{x u}\right]$ in (17). As a result, once $\Theta$ is obtained, there is no need to recalculate $\Theta$ in future learning iterations. Thus, the computational complexity per iteration in Algorithm 2 for some systems may be much lower than the computational complexity per iteration in Jiang and Jiang (2012, Algorithm 1).

Theorem 4.2 If (18) is satisfied, then $\lim _{k \rightarrow \infty} P_{k}=P^{*}$, and $\lim _{k \rightarrow \infty} K_{k}=K^{*}$, where $\left\{P_{k}\right\}_{k=0}^{\infty}$ and $\left\{K_{k}\right\}_{k=0}^{\infty}$ are obtained from Algorithm 2.

PROOF. If (18) is satisfied, (17) has unique solution. Then, the solution pair $\left(K_{k}, H_{k}\right)$ must satisfy (14), and therefore, $\tilde{P}_{k+1}$ and $P_{k+1}$ are equivalent to the ones in Algorithm 1. Otherwise, there exists a different pair $\left(K_{k}^{\prime}, H_{k}^{\prime}\right)$ satisfying (14), and is also the solution to (17). By Theorem 3.3, $\lim _{k \rightarrow \infty} P_{k}=P^{*}$. By continuity, $\lim _{k \rightarrow \infty} K_{k}=K^{*}$. This completes the proof.

Remark 4.3 Since the solution to (2) may diverge under the initial input $u$, it is necessary to design an upper bound $C>|x(0)|$, such that the system is stopped at $\tau>0$ whenever $|x(\tau)|>C$. This $C$, usually determined 


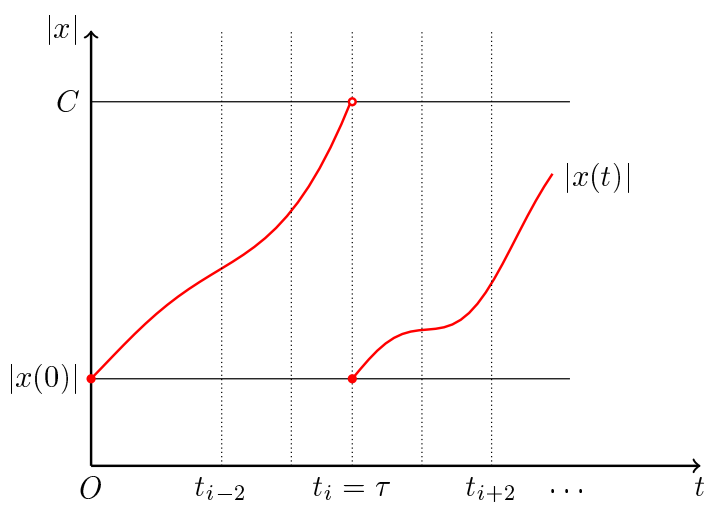

Fig. 1. Bounded learning trajectory.

by the physical restriction of the system, is the largest value of $|x(t)|$ that the system can tolerate. Then, we reset the state of the system to $x(0)$, and restart the system. Assign $t_{i}=\tau$ for some $0<i \leq l, x\left(t_{i}^{-}\right)=x(\tau)$, and $x\left(t_{i}^{+}\right)=x(0)$. Note that the trajectories after $t_{i}$ are not the same as the trajectories before $t_{i}$, due to the presence of the exploration noise $\omega$. See Fig. 1 for the details.

Remark 4.4 It can be easily seen from Corollary 3.9 that by choosing $Q>\bar{\varepsilon} I_{n}$, the control law $u=-K_{k} x$ obtained from Algorithm 2 is stabilizing for system (2).

Remark 4.5 Similar to Corollary 3.10, Algorithm 2 can also be used to solve the policy evaluation steps in Vrabie et al. (2009) and Jiang and Jiang (2012).

\section{Robust optimal design for linear stochastic systems}

In this section, we briefly mention an extension of Algorithm 2 to robust optimal control design for linear stochastic systems with input-dependent noise.

Consider the following linear stochastic system:

$$
d x=A x d t+B d v
$$

where $d v=\sum_{i=1}^{q} G_{i} u d w_{i}+u d t, \sum_{i=1}^{q} G_{i} u d w_{i}$ represents the input-dependent noise received by the plant from the input channel; $A, B, x, u$ satisfy the same conditions as in system (2); $w_{i}$ are independent Wiener processes (Arnold, 1974, Example (2.3.4)); and $G_{i} \in \mathbb{R}^{m \times m}$ are unknown constant matrices.

In this section, we aim at solving the optimal control problem described in Section 2.2, while using the online data generated from system (20). In the presence of the input-dependent noise $\sum_{i=1}^{q} G_{i} u d w_{i}$, the closedloop system under $u=-K^{*} x$ is GAS at the origin in mean square, if all the eigenvalues of

$$
\begin{array}{r}
\left(A-B K^{*}\right) \otimes I_{n}+I_{n} \otimes\left(A-B K^{*}\right) \\
+\sum_{i=1}^{q} B G_{i} K^{*} \otimes B G_{i} K^{*}
\end{array}
$$

are in the open left-half complex plane (Kleinman, 1969).

Note that the above condition holds if $\max _{i}\left|G_{i}\right|$ is small enough. In this case, the control law $u=-K^{*} x$ is called robust optimal, i.e., it is optimal in the absence of the noise $\sum_{i=1}^{q} G_{i} u d w_{i}$, and is robustly stabilizing in the presence of $\sum_{i=1}^{q} G_{i} u d w_{i}$.

Next, we derive the ADP algorithm. Given a Borel measurable locally essentially bounded input $u$ such that (20) has a unique and almost surely continuous solution, similar to (14), we have along the solutions to (20) that

$$
d\left(x^{T} P_{k} x\right)=x^{T} H_{k} x d t+u^{T} \Sigma_{k} u d t+2 x^{T} K_{k}^{T} R d v,
$$

where $\Sigma_{k}=\sum_{i=1}^{q} G_{i}^{T} B^{T} P_{k} B G_{i}$.

Following the notations in Section 4, define two matrices:

$$
\begin{aligned}
I_{x v} & =\left[\int_{t_{0}}^{t_{1}} x \otimes R d v, \int_{t_{1}}^{t_{2}} x \otimes R d v, \cdots, \int_{t_{l-1}}^{t_{l}} x \otimes R d v\right]^{T}, \\
I_{u u} & =\left[\int_{t_{0}}^{t_{1}} \bar{u} d s, \int_{t_{1}}^{t_{2}} \bar{u} d s, \cdots, \int_{t_{l-1}}^{t_{l}} \bar{u} d s\right]^{T},
\end{aligned}
$$

with $\bar{u}$ defined as in (16).

Then, we easily have

$$
\left[I_{x x}, 2 I_{x v}, I_{u u}\right]\left[\begin{array}{c}
\operatorname{vecs}\left(H_{k}\right) \\
\operatorname{vec}\left(K_{k}\right) \\
\operatorname{vecs}\left(\Sigma_{k}\right)
\end{array}\right]=\delta_{x x} \operatorname{vec}\left(P_{k}\right)
$$

If

$$
\operatorname{rank}\left(\left[I_{x x}, 2 I_{x v}, I_{u u}\right]\right)=\frac{n(n+1)}{2}+n m+\frac{m(m+1)}{2},
$$

then (22) has unique solutions of $H_{k}, K_{k}$, and $\Sigma_{k}$, and one easily has

$$
\left[\begin{array}{c}
\operatorname{vecs}\left(H_{k}\right) \\
\operatorname{vec}\left(K_{k}\right) \\
\operatorname{vecs}\left(\Sigma_{k}\right)
\end{array}\right]=\Pi \operatorname{vec}\left(P_{k}\right)
$$

where $\Pi=\left[I_{x x}, 2 I_{x v}, I_{u u}\right]^{\dagger} \delta_{x x}$. 


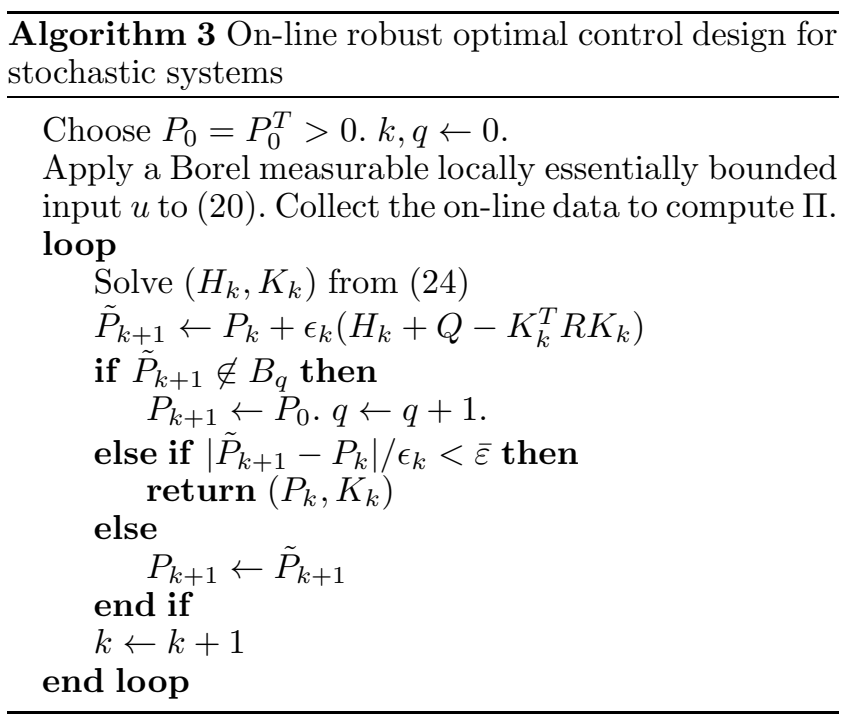

The VI-based ADP algorithm for system (20) is given in Algorithm 3.

The convergence analysis of Algorithm 3 is given in the following theorem. The proof of Theorem 5.1 is similar to the proof of Theorem 4.2, and thus is omitted.

Theorem 5.1 If (23) is satisfied, then $\lim _{k \rightarrow \infty} P_{k}=P^{*}$, and $\lim _{k \rightarrow \infty} K_{k}=K^{*}$, where $\left\{P_{k}\right\}_{k=0}^{\infty}$ and $\left\{K_{k}\right\}_{k=0}^{\infty}$ are obtained from Algorithm 3.

Remark 5.2 Since $\lim _{k \rightarrow \infty} K_{k}=K^{*}$, by continuity, there exists a small enough $\bar{\varepsilon}>0$, such that the eigenvalues of

$$
\begin{array}{r}
\left(A-B \hat{K}^{*}\right) \otimes I_{n}+I_{n} \otimes\left(A-B \hat{K}^{*}\right) \\
+\sum_{i=1}^{q} B G_{i} \hat{K}^{*} \otimes B G_{i} \hat{K}^{*},
\end{array}
$$

where $\hat{K}^{*}$ is obtained from Algorithm 3, are in the open left-half complex plane. Thus, the control law $u=-\hat{K}^{*} x$ is a robust stabilizer for system (2).

Remark 5.3 Note that compared with other continuoustime stochastic ADP methods (Jiang and Jiang, 2011, 2014a; Bian and Jiang, 2015) or stochastic adaptive control methods (Kumar, 1983; Duncan et al., 1999), Algorithm 3 is an on-line, off-policy algorithm that does not identify the system model, nor requires an initial stabilizing control policy.

\section{Illustrative examples}

\subsection{Application to power system control}

In this example, Algorithm 2 is used to develop a statefeedback controller for a single machine-infinite bus power system via on-line information. Consider the following linearized model (Wang et al., 1993):

$$
\begin{aligned}
\dot{\delta} & =\omega \\
\dot{\omega} & =-\frac{D}{2 H} \omega+\frac{\omega_{0}}{2 H}\left(P_{m 0}-P_{e}\right), \\
\dot{P}_{e} & =-\frac{1}{T_{d 0}^{\prime}} P_{e}+\frac{1}{T_{d 0}^{\prime}} v_{f}
\end{aligned}
$$

where $\delta$ is the power angle of the generator; $\omega$ is the relative speed of the generator; $P_{e}$ is the active electrical power delivered by the generator; and $P_{m}$ is the mechanical input power. The parameters are chosen as $D=5$ p.u., $H=4.0 \mathrm{~s}, \omega_{0}=314.159 \mathrm{rad} / \mathrm{s}$, and $T_{d 0}^{\prime}=2.4232 \mathrm{~s}$. The operating point is $\delta_{0}=47^{\circ}$ and $P_{m 0}=0.45 \mathrm{p} . \mathrm{u}$.

Then, we can write the error system in the form of (2), where

$$
\begin{array}{rlr}
x & =\left[\delta-\delta_{0}, \omega, P_{e}-P_{m 0}\right]^{T}, & u=v_{f}-P_{m 0}, \\
A & =\left[\begin{array}{ccc}
0 & 1 & 0 \\
0 & -\frac{D}{2 H} & -\frac{\omega_{0}}{2 H} \\
0 & 0 & -\frac{1}{T_{d 0}^{\prime}}
\end{array}\right], & B=\left[0,0, \frac{1}{T_{d 0}^{\prime}}\right]^{T} .
\end{array}
$$

Choose the weighting matrices in the cost as $Q=$ $\operatorname{diag}\{0.5,0.1,0.01\}$ and $R=1$.

Suppose there is an impulse disturbance at $t=0$, and thus $x(0)=[0,0,0.01]^{T}$. The on-line data from $t=0$ to $0.5 \mathrm{~s}$ are collected to apply Algorithm 2. We choose

$$
B_{q}=\left\{P \in \mathscr{S}_{+}^{3}:|P| \leq 10(q+1)\right\}, \quad \forall q=0,1, \cdots,
$$

$\epsilon_{k}=(k+1)^{-1}, \bar{\varepsilon}=10^{-3}$, and $P_{0}=0.01 I_{3}$. An approximated solution $\left(\hat{P}^{*}, \hat{K}^{*}\right)$ is obtained. The obtained control policy is applied to the system at $t=1.5 \mathrm{~s}$. The system trajectories are given in Fig. 2.

To further compare the results, we solve the optimal solution directly. The optimal values and the values obtained from Algorithm 2 are given below:

$$
\begin{aligned}
& P^{*}=\left[\begin{array}{ccc}
0.5142850 & 0.1870906 & -1.713461 \\
0.1870906 & 0.1267213 & -1.361699 \\
-1.7134612 & -1.3616993 & 22.754481
\end{array}\right], \\
& K^{*}=[-0.7071068,-0.5619426,9.390261] \text {, } \\
& \hat{P}^{*}=\left[\begin{array}{ccc}
0.5142820 & 0.1870922 & -1.713413 \\
0.1870922 & 0.1267241 & -1.361702 \\
-1.7134128 & -1.3617015 & 22.753929
\end{array}\right], \\
& \hat{K}^{*}=[-0.7070895,-0.5619445,9.390366] \text {. }
\end{aligned}
$$



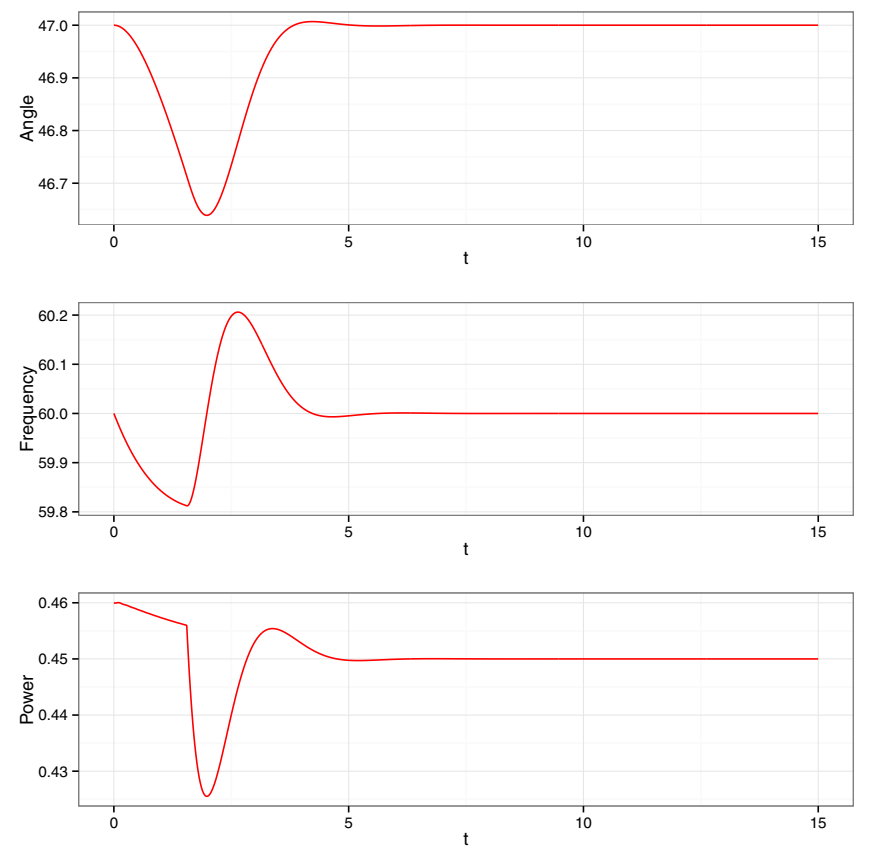

Fig. 2. Trajectories of the generator.

Table 1

Error for different initial values.

\begin{tabular}{|c|c|c|c|}
\hline$P_{0}$ & $0.01 I_{3}$ & $0.1 I_{3}$ & $I_{3}$ \\
\hline$\left|\hat{P}^{*}-P^{*}\right|$ & $3.57 \times 10^{-4}$ & $9.43 \times 10^{-4}$ & $6.10 \times 10^{-4}$ \\
\hline CPU time & $0.935 \mathrm{~s}$ & $1.098 \mathrm{~s}$ & $3.382 \mathrm{~s}$ \\
\hline \# iterations & 2778 & 3247 & 10094 \\
\hline
\end{tabular}

Obviously, $\hat{P}^{*}$ and $\hat{K}^{*}$ are quite close to $P^{*}$ and $K^{*}$, respectively.

Next, to show the convergence of Algorithm 2 under arbitrary initial value, we pick different $P_{0}$, and re-run the simulation program. The simulation results are provided in Table 1. The error plot of $\left|P_{k}-P^{*}\right|$ for different initial values is given in Fig. 3. CPU time is the computation time used in the ADP algorithm. This study was carried out using the 32-bit $\mathrm{R}$ program version 3.2 .2 , on a Linux machine (opensuse 13.1) with Intel(R) Core(TM) i7-4770K CPU @ 3.50GHz. Note that choosing $P_{0}=I_{3}$ causes the sequence $\left\{\tilde{P}_{k}\right\}_{k=0}^{\infty}$ more likely to escape from $B_{q}$ at the beginning of the learning process, and as a result requires longer computational time.

\subsection{Human arm movement in a divergent field}

In this example, we use the ADP method proposed in Section 5 to study the learning mechanism of human arm movements in a divergent field (DF). The model of the human arm motor system is given as follows (Liu and

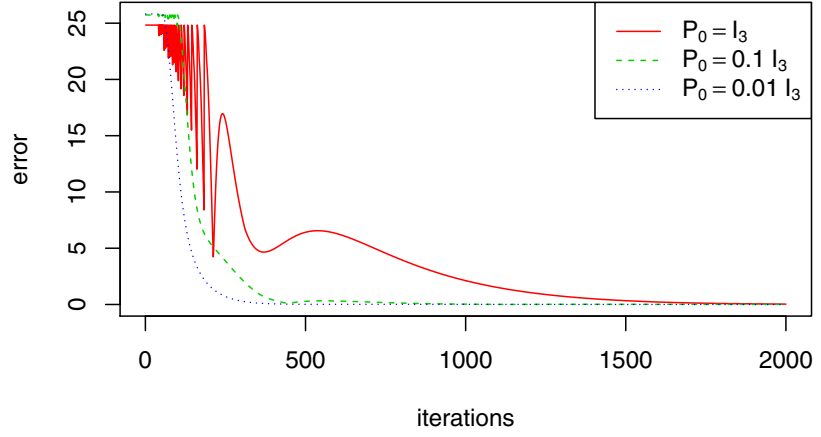

Fig. 3. Error plot of $\left|P_{k}-P^{*}\right|$ for different values of $P_{0}$.

Table 2

Parameters of the arm movement model

\begin{tabular}{ccc}
\hline Parameters & Description & Value \\
\hline$m$ & Hand mass & $1.3 \mathrm{~kg}$ \\
$b$ & Viscosity constant & $10 \mathrm{~N} \cdot \mathrm{s} / \mathrm{m}$ \\
$\tau$ & Time constant & $0.05 \mathrm{~s}$ \\
$c_{1}$ & Noise magnitude & 0.075 \\
$c_{2}$ & Noise magnitude & 0.025 \\
$\beta$ & force magnitude & 150 \\
\hline
\end{tabular}

Todorov, 2007; Jiang and Jiang, 2014a):

$$
\begin{aligned}
d p & =v d t, \\
m d v & =(a-b v+f) d t, \\
\tau d a & =(u-a) d t+d \zeta,
\end{aligned}
$$

where $p=\left[\begin{array}{ll}p_{x} & p_{y}\end{array}\right]^{T}, v=\left[\begin{array}{ll}v_{x} & v_{y}\end{array}\right]^{T}, a=\left[\begin{array}{ll}a_{x} & a_{y}\end{array}\right]^{T}$ denote the distance between the hand position and the origin, the hand velocity, and the actuator state, respectively; $u=\left[\begin{array}{ll}u_{x} & u_{y}\end{array}\right]^{T} \in \mathbb{R}^{2}$ is the control input; $f=\beta p_{x}$ is the force generated by the DF; and $d \zeta$ is the input-dependent noise (Liu and Todorov, 2007):

$$
d \zeta=\left[\begin{array}{ll}
c_{1} & 0 \\
c_{2} & 0
\end{array}\right]\left[\begin{array}{l}
u_{x} \\
u_{y}
\end{array}\right] d w_{1}+\left[\begin{array}{cc}
0 & -c_{2} \\
0 & c_{1}
\end{array}\right]\left[\begin{array}{l}
u_{x} \\
u_{y}
\end{array}\right] d w_{2},
$$

where $w_{1}$ and $w_{2}$ are independent Weiner processes. Then, we can rewrite the arm movement model in the form of (20), with $x=\left[p^{T}, v^{T}, a^{T}\right]^{T}$.

For comparison purpose, we choose the same system parameters as in Jiang and Jiang (2014a). The values of the parameters are provided in Table 2 . The weighting matrices in the cost are chosen as Jiang and Jiang (2014a, Eqs. (22) and (23))

When the human subject is first exposed to the DF, the variations are amplified by the divergence force, and thus 
the system is no longer stable (Fig. 4). Then, Algorithm 3 is used to improve the control policy. The simulation result of the sensorimotor system after 50 learning trials is given in Fig. 5. Interestingly, this result is consistent with the experimental results in Burdet et al. (2001); Franklin et al. (2003); Liu and Todorov (2007).

Different from the existing sensorimotor learning and control theory, which assumes that the central nervous system (CNS) can precisely know the system model via the system identification technique (Shadmehr and Mussa-Ivaldi, 2012, Chapter 9), our methodology is a non-model-based approach, and the optimal control is derived directly from real-time sensory data through repeated learning. Moreover, compared with the result reported in Jiang and Jiang (2014a), an initial stabilizing control policy is no longer required in our method. We conjecture that the proposed ADP method appears to be a suitable candidate for computational learning mechanism in the CNS to coordinate movements in unstable environments.

\subsection{Comparison between VI and PI}

As we have noted in the introduction and Remark 3.2, Algorithm 1 sometimes is more efficient than Kleinman's algorithm in the sense that (1) an initial stabilizing policy is not required, so the time in searching for an initial admissible controller as in the PI methods is reduced; and (2) there is no need to solve a Lyapunov equation in each iteration.

Next, we give an example to show that for some systems, if $\left\{\epsilon_{k}\right\}$ and $\left\{B_{q}\right\}$ are chosen properly, Algorithm 1 solves the optimal control problem with less computational time.

Consider a LQR problem with $A$ and $B$ given in Section 6.2 , and

$$
Q=\left[\begin{array}{cccccc}
1000 & 100 & 0 & 0 & 0 & 0 \\
100 & 1000 & 0 & 0 & 0 & 0 \\
0 & 0 & 20 & 1 & 0 & 0 \\
0 & 0 & 1 & 20 & 0 & 0 \\
0 & 0 & 0 & 0 & 0.01 & 0 \\
0 & 0 & 0 & 0 & 0 & 0.01
\end{array}\right], \quad R=0.01 I_{2}
$$

For simulation purpose only, the parameters in VI algorithm are chosen as $P_{0}=0.28 P^{*}, \epsilon_{k}=\left(k^{0.5}+40\right)^{-1}$, and

$$
B_{q}=\left\{P \in \mathscr{S}_{+}^{6}:|P| \leq(q+1) \times 10^{3}\right\}, \quad \forall q=0,1, \cdots,
$$

where $P^{*}$ is the optimal solution directly obtained by solving ARE. The initial feedback gain matrix in PI is
Table 3

Comparison between VI and PI.

\begin{tabular}{|c|c|c|}
\hline Algorithms & PI & VI \\
\hline$\left|\hat{P}^{*}-P^{*}\right|$ & 0.01762988 & 0.01211481 \\
\hline$\#$ iterations & 10 & 27 \\
\hline Total CPU time & $0.013 \mathrm{~s}$ & $0.011 \mathrm{~s}$ \\
\hline
\end{tabular}

chosen as $K_{0}=R^{-1} B^{T} P_{0}$. Note that $A-B K_{0}$ is Hurwitz.

The simulation results are given in Table 3 . In PI, the matrix inverse is calculated numerically through singular value decomposition.

Note that although the VI algorithm requires a large number of iterations, the computation time of VI is still less than the computation time of PI. This is because the computational complexity in each VI iteration is much lower, as there is no need to calculate matrix inverse. This example shows that for some LQR problems, our continuous-time VI may outperform Kleinman's algorithm in the sense that not only an initial stabilizing control gain matrix is not required, but also less computation time is required.

\section{Conclusions}

Previously obtained ADP results for continuous-time, continuous-state space systems are mainly based on PI methods. In this paper, we have developed for the first time a continuous-time VI algorithm. The convergence analysis for the proposed method is drawn on the basis of stochastic approximation theory. Moreover, we have also discussed the convergence issue of Vrabie's VI, or more precisely modified PI algorithm (Vrabie et al., 2013), and explained why this algorithm might fail to converge starting from any real symmetric and positive definite matrix. Two VI-based ADP methods have been developed to tackle the optimal control problem and the stochastic robust optimal control problem for linear continuous-time systems, respectively. Different from existing results, the knowledge of an admissible initial control policy is no longer required. Finally, we have validated our results with two simulation examples, all of which show that our method serves as a powerful tool to solve non-model-based, data-driven adaptive optimal control problems. Other emerging control applications of the proposed VI method include intelligent transportation systems (Liu et al., 2016; Gao et al., 2016).

\section{References}

Abounadi, J., Bertsekas, D. P., Borkar, V. S., 2002. Stochastic approximation for nonexpansive maps: Application to Q-learning algorithms. SIAM Journal on Control and Optimization 41 (1), 1-22. 


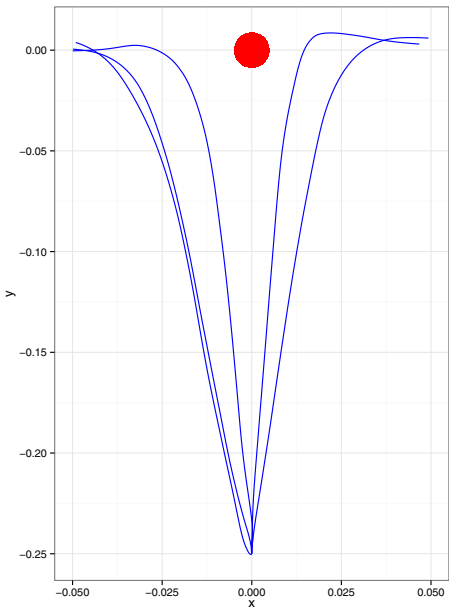

(a) Movement trajectories.
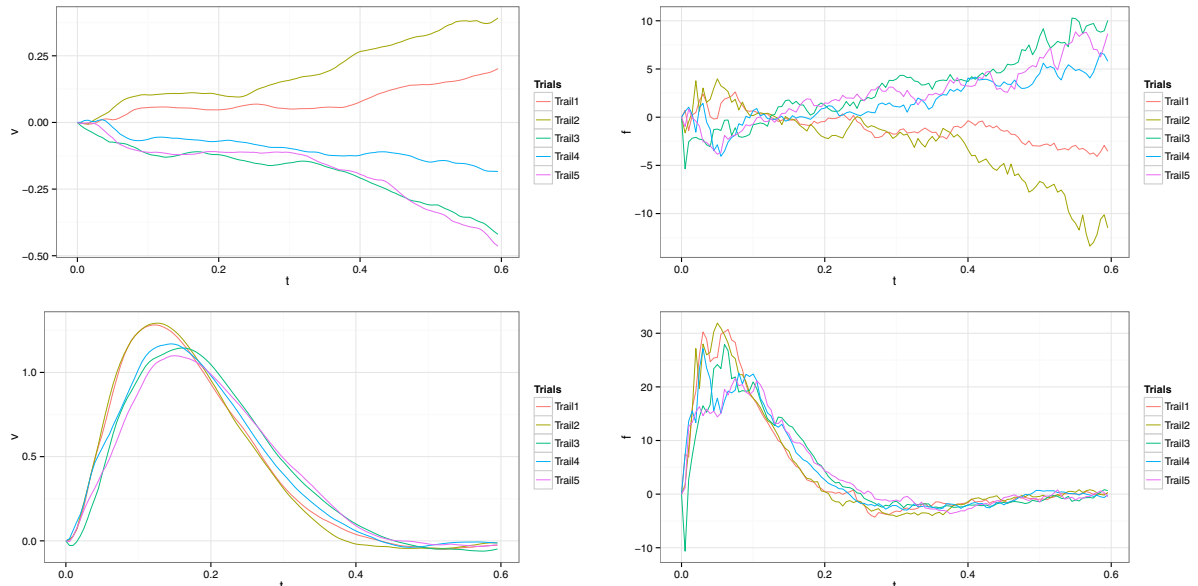

(b) Velocity and force curves.

Fig. 4. Five independent trajectories of the subject when first exposed to the DF.

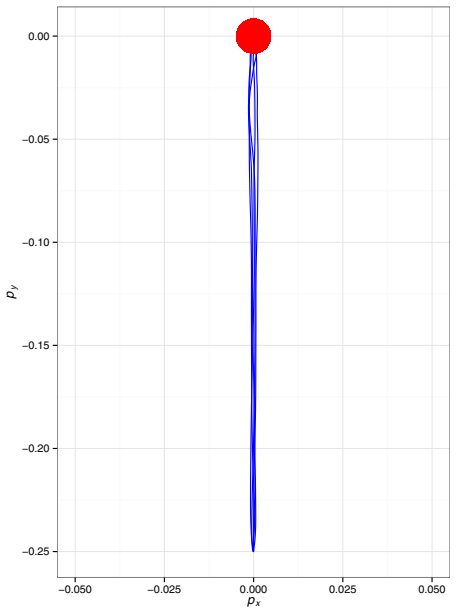

(a) Movement trajectories.
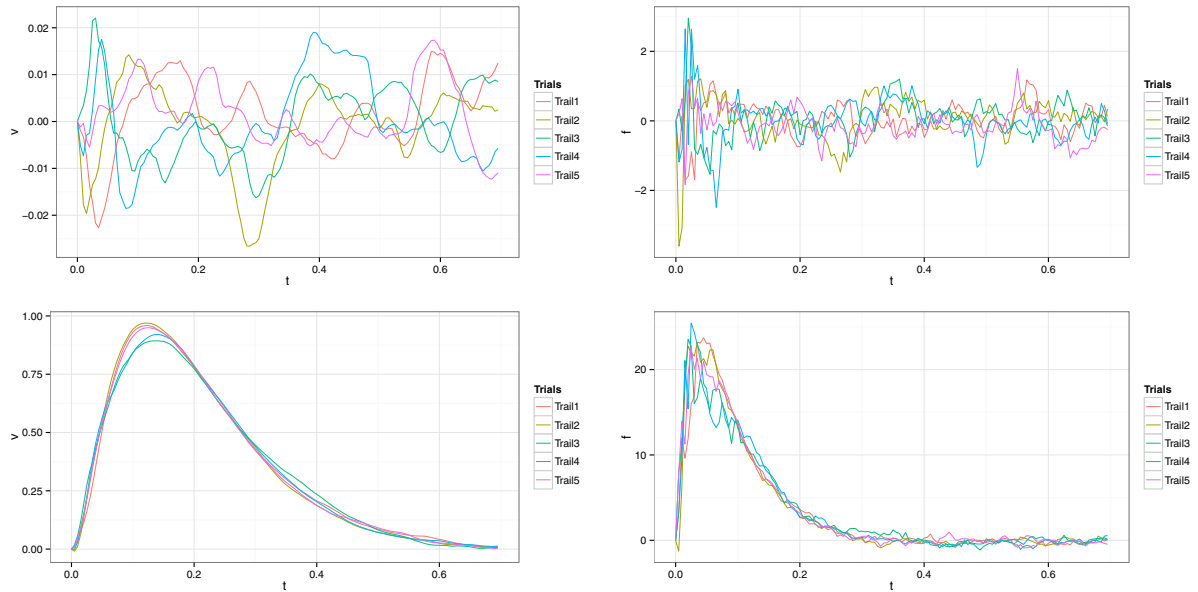

(b) Velocity and force curves.

Fig. 5. Five independent trajectories in DF after 50 learning trials.

Andrieu, C., Moulines, É., Priouret, P., 2005. Stability of stochastic approximation under verifiable conditions. SIAM Journal on Control and Optimization 44 (1), $283-312$.

Arnold, L., 1974. Stochastic Differential Equations: Theory and Applications. John Wiley \& Sons, Inc., New York.

Åström, K. J., Wittenmark, B., 1997. Adaptive Control, 2nd Edition. Addison-Wesley, Reading, MA.

Barto, A. G., Sutton, R. S., Anderson, C. W., 1983. Neuronlike adaptive elements that can solve difficult learning control problems. IEEE Transactions on Systems, Man and Cybernetics SMC-13 (5), 834-846.

Beard, R. W., Saridis, G. N., Wen, J. T., 1997. Galerkin approximations of the generalized Hamilton-JacobiBellman equation. Automatica 33 (12), 2159 - 2177.

Bellman, R. E., 1957. Dynamic Programming. Princeton
University Press, Princeton, NJ.

Bellman, R. E., 1961. Adaptive Control Processes: A Guided Tour. Princeton University Press, Princeton, NJ.

Bertsekas, D. P., 2005. Dynamic Programming and Optimal Control, 3rd Edition. Vol. 1. Athena Scientific, Belmont, MA.

Bertsekas, D. P., 2007. Dynamic Programming and Optimal Control, 3rd Edition. Vol. 2. Athena Scientific, Belmont, MA.

Bertsekas, D. P., 2011. Approximate policy iteration: a survey and some new methods. Journal of Control Theory and Applications 9 (3), 310-335.

Bertsekas, D. P., 2013. Abstract Dynamic Programming. Athena Scientific, Belmont, MA.

Bertsekas, D. P., Tsitsiklis, J. N., 1996. Neuro-Dynamic Programming. Athena Scientific, Belmont, MA. 
Bian, T., Jiang, Y., Jiang, Z. P., 2014. Adaptive dynamic programming and optimal control of nonlinear nonaffine systems. Automatica 50 (10), 2624-2632.

Bian, T., Jiang, Y., Jiang, Z. P., 2015. Decentralized adaptive optimal control of large-scale systems with application to power systems. IEEE Transactions on Industrial Electronics 62 (4), 2439-2447.

Bian, T., Jiang, Y., Jiang, Z. P., 2016. Adaptive dynamic programming for stochastic systems with state and control dependent noise. IEEE Transactions on Automatic Control, accepted.

Bian, T., Jiang, Z. P., 2015. Adaptive optimal control for linear stochastic systems with additive noise. In: Proceedings of the 34th Chinese Control Conference (CCC). pp. 3011-3016.

Billingsley, P., 2009. Convergence of Probability Measures, 2nd Edition. John Wiley \& Sons, Inc., New York, NY.

Borkar, V. S., 1998. Asynchronous stochastic approximations. SIAM Journal on Control and Optimization $36(3), 840-851$.

Burdet, E., Osu, R., Franklin, D. W., Milner, T. E., Kawato, M., 2001. The central nervous system stabilizes unstable dynamics by learning optimal impedance. Nature 414 (6862), 446-449.

Chen, H.-F., 2002. Stochastic Approximation and Its Applications. Springer US.

Chen, H.-F., Zhu, Y., 1986. Stochastic approximation procedures with randomly varying truncations. Scientia Sinica (Series A) XXIX (9), 914-926.

Duncan, T. E., Guo, L., Pasik-Duncan, B., 1999. Adaptive continuous-time linear quadratic Gaussian control. IEEE Transactions on Automatic Control 44 (9), 1653-1662.

Franklin, D. W., Burdet, E., Osu, R., Kawato, M., Milner, T., 2003. Functional significance of stiffness in adaptation of multijoint arm movements to stable and unstable dynamics. Experimental Brain Research 151 (2), 145-157.

Gao, W., Jiang, Z. P., Ozbay, K., 2016. Data-driven adaptive optimal control of connected vehicles. IEEE Transactions on Intelligent Transportation Systems, under review.

Heydari, A., 2014. Revisiting approximate dynamic programming and its convergence. IEEE Transactions on Cybernetics 44 (12), 2733-2743.

Jeung, E. T., Oh, D. C., Kim, J. H., Park, H. B., 1996. Robust controller design for uncertain systems with time delays: LMI approach. Automatica 32 (8), 1229 $-1231$.

Jiang, Y., Jiang, Z. P., 2011. Approximate dynamic programming for optimal stationary control with controldependent noise. IEEE Transactions on Neural Networks 22 (12), 2392-2398.

Jiang, Y., Jiang, Z. P., 2012. Computational adaptive optimal control for continuous-time linear systems with completely unknown dynamics. Automatica 48 (10), $2699-2704$

Jiang, Y., Jiang, Z. P., 2014a. Adaptive dynamic pro- gramming as a theory of sensorimotor control. Biological Cybernetics 108 (4), 459-473.

Jiang, Y., Jiang, Z. P., 2014b. Robust adaptive dynamic programming and feedback stabilization of nonlinear systems. IEEE Transactions on Neural Networks and Learning Systems 25 (5), 882-893.

Jiang, Z. P., Jiang, Y., 2013. Robust adaptive dynamic programming for linear and nonlinear systems: An overview. European Journal of Control 19 (5), 417 425.

Kantorovich, L., Akilov, G., 1982. Functional Analysis, 2nd Edition. Pergamon Press, Elmsford, NY.

Khalil, H. K., 2002. Nonlinear Systems, 3rd Edition. Prentice Hall, Upper Saddle River, NJ.

Khargonekar, P. P., Petersen, I. R., Zhou, K., 1990. Robust stabilization of uncertain linear systems: quadratic stabilizability and $H^{\infty}$ control theory. IEEE Transactions on Automatic Control 35 (3), 356-361.

Kleinman, D. L., 1968. On an iterative technique for Riccati equation computations. IEEE Transactions on Automatic Control 13 (1), 114-115.

Kleinman, D. L., 1969. On the stability of linear stochastic systems. IEEE Transactions on Automatic Control 14 (4), 429-430.

Kučera, V., 1973. A review of the matrix Riccati equation. Kybernetika 9 (1), 42-61.

Kumar, P. R., 1983. Optimal adaptive control of linearquadratic-Gaussian systems. SIAM Journal on Control and Optimization 21 (2), 163-178.

Kushner, H. J., Yin, G. G., 2003. Stochastic Approximation and Recursive Algorithms and Applications. Springer New York.

Lancaster, P., Rodman, L., 1995. Algebraic Riccati Equations. Oxford University Press, New York.

Leake, R., Liu, R., 1967. Construction of suboptimal control sequences. SIAM Journal on Control 5 (1), 5463.

Lewis, F. L., Vamvoudakis, K. G., 2011. Reinforcement learning for partially observable dynamic processes: Adaptive dynamic programming using measured output data. IEEE Transactions on Systems, Man, and Cybernetics, Part B: Cybernetics 41 (1), 14-25.

Lewis, F. L., Vrabie, D., 2009. Reinforcement learning and adaptive dynamic programming for feedback control. IEEE Circuits and Systems Magazine 9 (3), 3250.

Lewis, F. L., Vrabie, D., Vamvoudakis, K. G., 2012. Reinforcement learning and feedback control: Using natural decision methods to design optimal adaptive controllers. IEEE Control Systems 32 (6), 76-105.

Li, H., Liu, D., Wang, D., 2014. Integral reinforcement learning for linear continuous-time zero-sum games with completely unknown dynamics. IEEE Transactions on Automation Science and Engineering 11 (3), $706-714$.

Liu, D., Todorov, E., 2007. Evidence for the flexible sensorimotor strategies predicted by optimal feedback control. The Journal of Neuroscience 27 (35), 93549368 . 
Liu, D., Wang, D., Zhao, D., Wei, Q., Jin, N., 2012. Neural-network-based optimal control for a class of unknown discrete-time nonlinear systems using globalized dual heuristic programming. IEEE Transactions on Automation Science and Engineering 9 (3), 628-634.

Liu, T., Lu, X., Jiang, Z. P., Jan 2016. A junctionby-junction feedback-based strategy with convergence analysis for dynamic traffic assignment. Science China Information Sciences 59 (1), 010203:1-010203:17.

Ljung, L., 1977. Analysis of recursive stochastic algorithms. IEEE Transactions on Automatic Control $22(4), 551-575$.

Modares, H., Lewis, F. L., 2014. Linear quadratic tracking control of partially-unknown continuous-time systems using reinforcement learning. IEEE Transactions on Automatic Control 59 (11), 3051-3056.

Murray, J. J., Cox, C. J., Lendaris, G. G., Saeks, R., 2002. Adaptive dynamic programming. IEEE Transactions on Systems, Man, and Cybernetics, Part C: Applications and Reviews 32 (2), 140-153.

Ni, Z., He, H., Zhong, X., Prokhorov, D. V., 2015. Modelfree dual heuristic dynamic programming. IEEE Transactions on Neural Networks and Learning Systems 26 (8), 1834-1839.

Prokhorov, D. V., Wunsch, D. C., 1997. Adaptive critic designs. IEEE Transactions on Neural Networks 8 (5), 997-1007.

Puterman, M. L., 1994. Markov Decision Processes: Discrete Stochastic Dynamic Programming. John Wiley \& Sons, Inc., New York, NY.

Reed, M., Simon, B., 1980. Methods of Modern Mathematical Physics: Functional analysis. Academic Press, San Diego.

Robbins, H., Monro, S., 1951. A stochastic approximation method. The Annals of Mathematical Statistics 22 (3), 400-407.

Rudin, W., 1987. Real and Complex Analysis. McGrawHill International, New York, NY.

Shadmehr, R., Mussa-Ivaldi, S., 2012. Biological Learning and Control: How the Brain Builds Representations, Predicts Events, and Makes Decisions. The MIT Press, Cambridge, MA.

Shayman, M., 1986. Phase portrait of the matrix Riccati equation. SIAM Journal on Control and Optimization 24 (1), 1-65.

Si, J., Barto, A. G., Powell, W. B., Wunsch, D. C. (Eds.), 2004. Handbook of Learning and Approximate Dynamic Programming. Wiley-IEEE Press, Piscataway, NJ.

Skorokhod, A. V., 1956. Limit theorems for stochastic processes. Theory of Probability \& Its Applications 1 (3), 261-290.

Song, R., Lewis, F. L., Wei, Q., Zhang, H., Jiang, Z. P., Levine, D., 2015. Multiple actor-critic structures for continuous-time optimal control using input-output data. IEEE Transactions on Neural Networks and Learning Systems 26 (4), 851-865.

Sutton, R. S., 1988. Learning to predict by the methods of temporal differences. Machine Learning 3 (1), 9-44.

Sutton, R. S., Barto, A. G., 1998. Reinforcement Learning: An Introduction. The MIT Press, Cambridge, MA.

Tao, G., 2014. Multivariable adaptive control: A survey. Automatica 50 (11), 2737-2764.

Tsitsiklis, J. N., 1994. Asynchronous stochastic approximation and Q-learning. Machine Learning 16 (3), 185202.

Vrabie, D., Pastravanu, O., Abu-Khalaf, M., Lewis, F. L., 2009. Adaptive optimal control for continuoustime linear systems based on policy iteration. Automatica 45 (2), 477-484.

Vrabie, D., Vamvoudakis, K. G., Lewis, F. L., 2013. Optimal Adaptive Control and Differential Games by Reinforcement Learning Principles. Vol. 81. Institution of Engineering and Technology, London, UK.

Wang, D., Liu, D., Wei, Q., Zhao, D., Jin, N., 2012. Optimal control of unknown nonaffine nonlinear discretetime systems based on adaptive dynamic programming. Automatica 48 (8), 1825-1832.

Wang, F.-Y., Jin, N., Liu, D., Wei, Q., 2011. Adaptive dynamic programming for finite-horizon optimal control of discrete-time nonlinear systems with $\varepsilon$-error bound. IEEE Transactions on Neural Networks 22 (1), $24-36$.

Wang, Y., Hill, D. J., Middleton, R. H., Gao, L., 1993. Transient stability enhancement and voltage regulation of power systems. IEEE Transactions on Power Systems 8 (2), 620-627.

Watkins, C. J. C. H., Dayan, P., 1992. Q-learning. Machine Learning 8 (3-4), 279-292.

Willems, J. L., 1971. Least squares stationary optimal control and the algebraic Riccati equation. IEEE Transactions on Automatic Control 16 (6), 621-634.

Wolpert, D. M., Diedrichsen, J., Flanagan, J. R., 2011. Principles of sensorimotor learning. Nature Reviews Neuroscience 12 (12), 739-751.

Xu, H., Jagannathan, S., Lewis, F. L., 2012. Stochastic optimal control of unknown linear networked control system in the presence of random delays and packet losses. Automatica 48 (6), 1017-1030.

Zargarzadeh, H., Dierks, T., Jagannathan, S., 2015. Optimal control of nonlinear continuous-time systems in strict-feedback form. IEEE Transactions on Neural Networks and Learning Systems 26 (10), 2535-2549.

Zhang, H., Luo, Y., Liu, D., 2009. Neural-networkbased near-optimal control for a class of discretetime affine nonlinear systems with control constraints. IEEE Transactions on Neural Networks 20 (9), 14901503.

Zhou, K., Khargonekar, P. P., 1988. Robust stabilization of linear systems with norm-bounded time-varying uncertainty. Systems \& Control Letters 10 (1), 17 - 20.

\section{A Proof of Lemma 3.4}

This proof contains three major steps. 
First, note that $\operatorname{vecs}(\cdot)$ is an isometric isomorphism ${ }^{1}$ from $\mathscr{S}^{q}$ to $\mathbb{R}^{q(q+1) / 2}$, for any integer $q>0$. Thus, one can rewrite (8) as the following ODE:

$$
\dot{p}=h(p),
$$

where $p=\operatorname{vecs}(P)$, and $h(\cdot): \mathbb{R}^{n(n+1) / 2} \rightarrow \mathbb{R}^{n(n+1) / 2}$ is defined as

$$
h(p)=\operatorname{vecs}\left(A^{T} P+P A-P B R^{-1} B^{T} P+Q\right) .
$$

It was shown in Kučera (1973, Theorem 17) that the solution to (8) converges to $P^{*}$ asymptotically, if $P(0) \in$ $\mathscr{S}_{+}^{n}$. Thus, $p^{*}=\operatorname{vecs}\left(P^{*}\right)$ is a locally asymptotically stable equilibrium of (A.1), and the region of attraction of $p^{*}$, denoted by $R_{A}$ (Khalil, 2002, Lemma 8.1), satisfies

$$
R_{A} \supset \operatorname{vecs}\left(\mathscr{S}_{+}^{n}\right)
$$

By converse Lyapunov theorem (Khalil, 2002, Theorem 4.17 ), we know there exists a smooth function $V(\cdot)$ : $R_{A} \rightarrow \mathbb{R}_{+}$, such that

$$
\begin{aligned}
\nabla V(p) h(p) & <0, \quad V(p)>0, \quad \forall p \in R_{A} \backslash\left\{p^{*}\right\} \\
\lim _{p \rightarrow \partial R_{A}} V(p) & =\infty, \quad \nabla V\left(p^{*}\right) h\left(p^{*}\right)=0, \quad V\left(p^{*}\right)=0 .
\end{aligned}
$$

As a result, $\{p: V(p) \leq C\}$ is a compact subset of $R_{A}$, for all $C>0$.

Second, we show that there exist a compact set $\mathcal{K} \subset \mathscr{S}_{+}^{n}$ and an integer $\bar{N} \geq 0$, such that if $N:=\inf \{k \geq \bar{N}$ : $\left.P_{k}=P_{0}\right\}<\infty$, then $\left\{P_{k}\right\}_{k=N}^{\infty} \subset \mathcal{K}$.

Suppose $P_{0} \neq P^{*}$. Then, there exists $C_{0}>0$, such that $C_{0}<V\left(p_{0}\right)$, where $p_{0}=\operatorname{vecs}\left(P_{0}\right)$. Moreover, since $p_{0} \in$ $R_{A}$, there exists $C_{1}>C_{0}$, such that $V\left(p_{0}\right)<C_{1}$. Choose

$$
\delta \in\left(0, \inf _{\left\{p: C_{0} \leq V(p) \leq C_{1}\right\}}|\nabla V(p) h(p)|\right) .
$$

Then, there exists $\bar{\epsilon}_{1}>0$, such that for all $0<\epsilon<\bar{\epsilon}_{1}$, $t \in[0,1]$, and $p \in\left\{p: C_{0} \leq V(p) \leq C_{1}\right\}$, we have $p+\epsilon \operatorname{th}(p) \in R_{A}$, and

$$
\leq \inf _{\left\{p: C_{0} \leq V(p) \leq C_{1}\right\}}|\nabla V(p) h(p)|-\delta .
$$

\footnotetext{
1 A bounded linear operator is called an isometric isomorphism if it is a norm preserving bijection which is continuous and has a continuous inverse (Reed and Simon, 1980, pp. 71).
}

This gives

$$
\begin{aligned}
& V(p+\epsilon h(p))-V(p) \\
= & \epsilon \nabla V(p) h(p)+\epsilon \int_{0}^{1}(\nabla V(p+\epsilon t h(p)) h(p)-\nabla V(p) h(p)) d t \\
\leq & -\epsilon \inf _{\left\{p: C_{0} \leq V(p) \leq C_{1}\right\}}|\nabla V(p) h(p)| \\
& \left.+\epsilon \inf _{\left\{p: C_{0} \leq V(p) \leq C_{1}\right\}}|\nabla V(p) h(p)|-\delta\right) \\
= & -\epsilon \delta .
\end{aligned}
$$

Therefore, for all $p \in\left\{p: C_{0} \leq V(p) \leq C_{1}\right\}, V(p+$ $\epsilon h(p))<V(p) \leq C_{1}$. On the other hand, if $V(p)<$ $C_{0}$, by continuity, there exists $\bar{\epsilon}_{2}>0$, such that $V(p+$ $\epsilon h(p))<C_{1}, \forall \epsilon<\bar{\epsilon}_{2}, \forall V(p)<C_{0}$.

Choose $\bar{N}$, such that $\epsilon_{k}<\min \left\{\bar{\epsilon}_{1}, \bar{\epsilon}_{2}\right\}$ for all $k \geq \bar{N}$. Denote $p_{N}=\operatorname{vecs}\left(P_{N}\right)$. Since $C_{0} \leq V\left(p_{N}\right)=V\left(p_{0}\right) \leq$ $C_{1}$, and $\lim _{q \rightarrow \infty} B_{q}=\mathscr{S}_{+}^{n}$, one has by induction that $P_{k} \in \mathcal{K}$ for all $k \geq N$, where

$$
\begin{aligned}
\mathcal{K} & =\bigcup_{q \in \mathbb{Z}_{+}}\left(B_{q} \cap\left\{P \in \mathscr{S}^{n}: V(\operatorname{vecs}(P)) \leq C_{1}\right\}\right) \\
& =\mathscr{S}_{+}^{n} \cap\left\{P: V(\operatorname{vecs}(P)) \leq C_{1}\right\} .
\end{aligned}
$$

Since vecs $(\cdot)$ has a continuous inverse, and $\{p: V(p) \leq$ $\left.C_{1}\right\}$ is compact, $\left\{P \in \mathscr{S}^{n}: V(\operatorname{vecs}(P)) \leq C_{1}\right\}$ is compact (Rudin, 1987, Theorem 2.10), and thus $\mathcal{K}$ is compact (Rudin, 1987, pp. 36, Corollary).

Finally, we show the conclusion of Lemma 3.4 holds. By contradiction, assume $\left\{P_{k}\right\}_{k=0}^{\infty}$ is unbounded. Thus there exists a subsequence $\left\{P_{k_{1}(i)}\right\}_{i=1}^{\infty}$ with $\left|P_{k_{1}(i)}\right|>i$, $i=1, \cdots$. This implies that $\left\{\tilde{P}_{k}\right\}_{k=0}^{\infty}$ in Algorithm 1 is also unbounded (Otherwise, $\left\{\tilde{P}_{k}\right\}_{k=0}^{\infty}$ remains in a $B_{q}$ for some $q \in \mathbb{Z}_{+}$, and thus $\left\{P_{k}\right\}_{k=0}^{\infty}$ also remains in $\left.B_{q}\right)$. Therefore, there exists a subsequence $\left\{P_{k_{2}(i)}\right\}_{i=1}^{\infty}$ satisfying $P_{k_{2}(i)}=P_{0}$ for all $i$. Define $\left\{P_{k^{\prime \prime}}\right\}=\left\{P_{k_{1}(i)}\right\} \cup$ $\left\{P_{k_{2}(i)}\right\}$. Obviously, $\left\{P_{k^{\prime \prime}}\right\}$ is unbounded, and visits $P_{0}$ infinitely often. However, this contradicts to the conclusion in the second part of this proof. This completes the proof. 